\title{
Subcellular sequencing of single neurons reveals the dendritic transcriptome of GABAergic interneurons.
}

Julio D. Perez¹, Susanne tom Dieck¹, Beatriz Alvarez-Castelao², Ivy C.W. Chan³, Erin M. Schuman ${ }^{1}$

${ }^{1}$ Max Planck Institute for Brain Research, Frankfurt am Main, Germany

2Department of Biochemistry and Molecular Biology, Veterinary School, Complutense University of Madrid, Madrid, Spain

${ }^{3}$ Department of Behavior and Brain Organization, Center of Advanced European Studies and Research, Bonn, Germany

\section{Abstract}

The localization and translation of mRNAs to dendrites and axons maintains and modifies the local proteome of neurons, and is essential for synaptic plasticity. Although significant efforts have allowed the identification of localized mRNAs in excitatory neurons, it is still unclear whether interneurons also localize a large population of mRNAs. In addition, the variability in the population of localized mRNAs within and between cell-types is unknown. Here we developed a method for the transcriptomic characterization of a single neuron's subcellular compartments, which combines laser capture microdissection with scRNAseq. This allowed us to separately profile the dendritic and somatic transcriptomes of individual rat hippocampal neurons and investigate the relation in mRNA abundances between the soma and dendrites of single glutamatergic and GABAergic neurons. We identified two types of glutamatergic and three types of GABAergic interneurons and we found that, like their excitatory counterparts, interneurons contain a rich repertoire of $\sim 4000$ mRNAs. The individual somatic transcriptomes exhibited more cell type-specific features than their associated dendritic transcriptomes. The detection and abundance of dendritic mRNAs was not always simply predicted by their somatic counterparts. Finally, using cell-type specific metabolic labelling of isolated neurites, we demonstrated that the processes not only of Glutamatergic but also of GABAergic neurons are capable of local translation, suggesting mRNA localization and local translation is a general property of neurons. 


\section{Introduction}

The synaptic connections between the dendritic and axonal processes of individual neurons provides the substrate for the flow, modification and storage of information in the brain. Because they usually extend hundreds of micrometers from their cell bodies, axons and dendrites must operate with some biochemical and electrical autonomy to accommodate changes at the speed of synaptic transmission. This is accomplished by post-translational modifications and regulation of the local proteome, including the local synthesis of proteins. By localizing specific mRNAs to these compartments, a neuron can control where and when different proteins are made and function (Holt et al., 2019). Working with tissue-derived RNA samples, multiple groups have identified transcripts present in dendrites and axons, revealing which proteins are likely generated by local translation (Cajigas et al., 2012; Glock et al., 2020.; Gumy et al., 2011; Hafner et al., 2019; Poon et al., 2006; Zhong et al., 2006). However, by design, these tissue-based approaches forgo fundamental features of local transcriptomes that can only be dissected at the level of individual neurons. First, how universal is the localization and translation of mRNAs in different neuronal cell-types? Second, how variable are the dendritic/axonal transcriptomes from one neuron type to another? Third, how much influence does the somatic abundance of a given mRNA have on its localization in neuronal processes?

It is unclear if subcellular mRNA localization and translation occurs in all types of neurons, and whether it varies according to cell type or cell state. The current state-of-the-art includes two kinds of sequencing studies. On one hand, bulk RNA studies of microdissected tissue (containing multiple cell types) have profiled mRNAs in the neurites (Poon et al., 2006) or an entire brain slice neuropil (Cajigas et al., 2012; Glock et al., 2020; Zhong et al., 2006) providing a comprehensive view of mRNAs localized to the neurite population. However, quantitative expression values obtained from these bulk sequencing approaches do not reflect the actual mRNA values present in an individual neuron, but rather the population average, potentially hiding intra-population heterogeneity in the local transcriptome. On the other hand, unbiased single cell RNA- 
seq (scRNA-seq) studies circumvent the averaging effect of bulk approaches and have revealed extensive diversity in cellular composition of the brain. However, these methods require the generation of cell suspensions, resulting in the shearing off of neuronal processes and the profiling of exclusively somatic mRNAs (Prakadan et al., 2017). In addition, most bulk-sequencing studies have attributed the identified mRNAs to excitatory neurons; it is still unclear whether local translation occurs in different classes of inhibitory interneurons. Indeed a better understanding of the subcellular transcriptome of these neurons may help explain their diverse size, transmitter phenotype, axonal and dendritic architecture, and electrophysiological properties (Huang and Paul, 2019; Pelkey et al., 2017). Moreover, the growing appreciation of the brain's cell type complexity and the fact that many of the variable genes act at the synapse (Saunders et al., 2018; Zeisel et al., 2018) suggest that specialization of dendritic and axonal compartments is a critical consequence of cell differentiation. Determining which mRNAs are locally translated could thus highlight unique functions and dynamics of cell-type specific compartments.

Single cell approaches that include dendrites or axons could, in principle, elucidate how different classes of neurons (excitatory, inhibitory, peptidergic, etc.) establish and maintain diverse transcriptomes across different compartments. Since it houses the source of transcription (the nucleus), the neuronal soma is the source of mRNAs from which local transcriptomes are generated. It is unclear, however, how much a transcript's somatic level influences its localization in processes, and whether transcripts can be enriched or excluded from the neurites regardless of their somatic abundances. In fact, mRNA localization is often defined based on either the absolute abundance in neurites or the relative enrichment in comparison to somata (Kügelgen and Chekulaeva, 2020). These absolute versus relative values can generate significantly different abundance rankings of the mRNAs that population local transcriptomes. For instance, a highly expressed somatic transcript that is also abundant in dendrites would be considered highly dendritic in absolute terms but less so in relative terms. The opposite can be true for a lowly expressed transcript frequently found in dendrites albeit at low copy numbers. These distinctions could be biologically meaningful: absolute values may indicate the 
number of molecules needed in a compartment (Kosik, 2016), while relative values may reflect regulation of mRNA concentrations between compartments (Liu et al., 2016). Estimates of these values based on bulk expression may be distorted since diversity among individual neurons in both the somatic and local transcriptomes is averaged out. Ideally, the transcriptome of each subcellular compartment would be measured and compared within each neuron. Such analyses could also elucidate the functions and thus the biochemical environments that distinguish each neuronal compartment.

Here we developed a method to profile the transcriptome of subcellular compartments of a single neuron, by combining the precision of laser capture microdissection (LCM) with the sensitivity of scRNA-seq. We used this method to identify the dendritic and somatic transcriptomes of cultured hippocampal neurons and observed mRNAs in the dendrites of all types of neurons including three GABAergic types. We found that dendritic transcriptomes were different from somatic transcriptomes and detected distinct patterns of dendritic mRNA variability according to cell type. Additionally, we describe the relation between somatic abundance and dendritic localization; we found that whether an mRNA is dendritically-enriched or de-enriched strongly correlates with its cellular function. Finally, we show that proteins are locally synthesized in the dendrites of both Glutamatergic and GABAergic neurons, suggesting mRNA localization and local translation is a general neuronal property.

\section{Results}

\section{Development of a subcellular scRNA-seq method}

To profile individually the somatic and dendritic transcriptome of a single neuron we used laser capture microdissection (LCM), previously used to profile the transcriptome of individual cells from tissue sections (Foley et al., 2019; Nichterwitz et al., 2016), since it allows for the dissection of biological material with $\mu \mathrm{m}$ resolution. To target subcellular structures with LCM, we first optimized the dissection of individual dendritic processes of rat primary cultured hippocampal neurons (see methods). To maximize sensitivity and throughput, we adapted a scRNA-seq protocol for droplet microfluidics (Macosko et al., 
2015) which tags mRNAs with both an index and unique molecule identifier (UMI) allowing sample pooling and processing followed by the identification and quantification of mRNAs from individual neuronal somata and dendritic arbors (Figure 1A). Using 92 synthetic RNA standards of different concentrations, we determined the method accurately measures RNA expression levels and reliably detects RNA species present in as low as 4 copies per sample (Figures S1A and S1B).

We thus generated a large dataset of dendritic arbors and somata from single neurons which were relatively isolated within the culture dish. Every neuron was first imaged, followed by capture of the soma, followed by collection of all accessible dendrites (Figure $\mathrm{S1C}$ ). Neurites of smaller diameters were avoided since they could be axons. We also collected additional somata from other neurons to expand the depth of cell-types explored. As a negative control, we laser-captured comparable areas within the dish with no visible cellular structure ("empty cuts"). All samples were then processed as described above (also see methods). Data processing steps and analyses of the sequencing data are summarized in Figure S1D. In total, we collected 795 samples: 276 dendritic samples and their respective somata, 227 somata without a dendritic arbor, and 16 empty cuts.

Somatic and dendritic samples contained significantly more RNA molecules and unique genes than empty cuts (Figure 1B and S1E, mean RNA counts= somata 68,004, dendrites 7,575, empty cuts 63), highlighting the accuracy of our laser-capture microdissection. On average we detected 3,828 unique transcripts in somata, 673 transcripts in dendrites, and 34 genes in empty cuts. Some cellular samples exhibited very low counts (somata and dendritic samples with less than 800 and 80 genes detected, respectively) and were thus not retained for downstream analyses (Figure S1E). We analyzed the depth of our subcellular transcriptomes and estimated that we identified the majority of genes expressed in the soma, whereas for dendrites, the most abundant dendritic genes were identified, but some less abundant genes were probably below our detection limit (Figure S1C). To identify potential glia or low-quality cells we performed uniform manifold approximation and projection (UMAP) for all samples, and identified two 

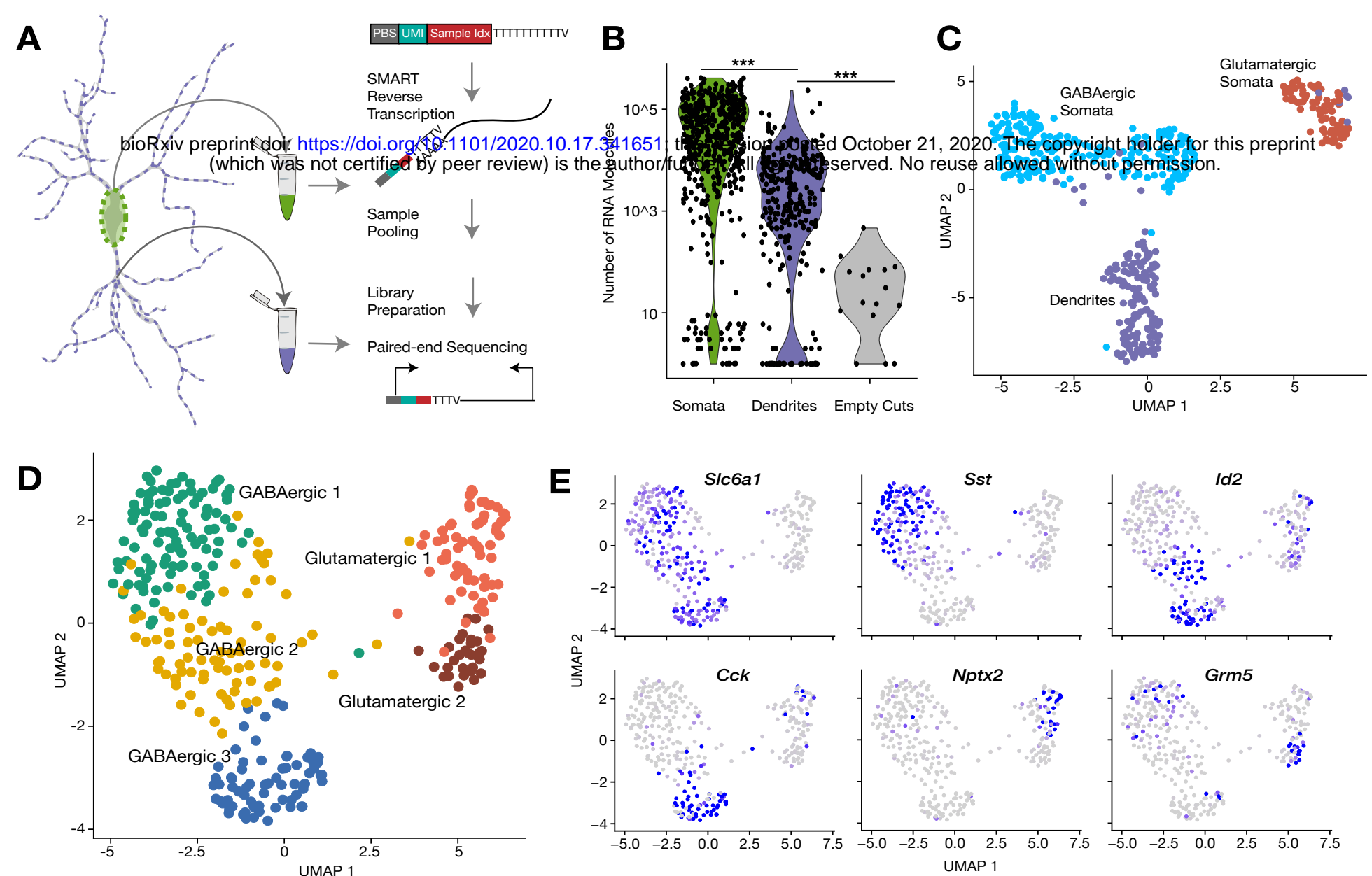

F

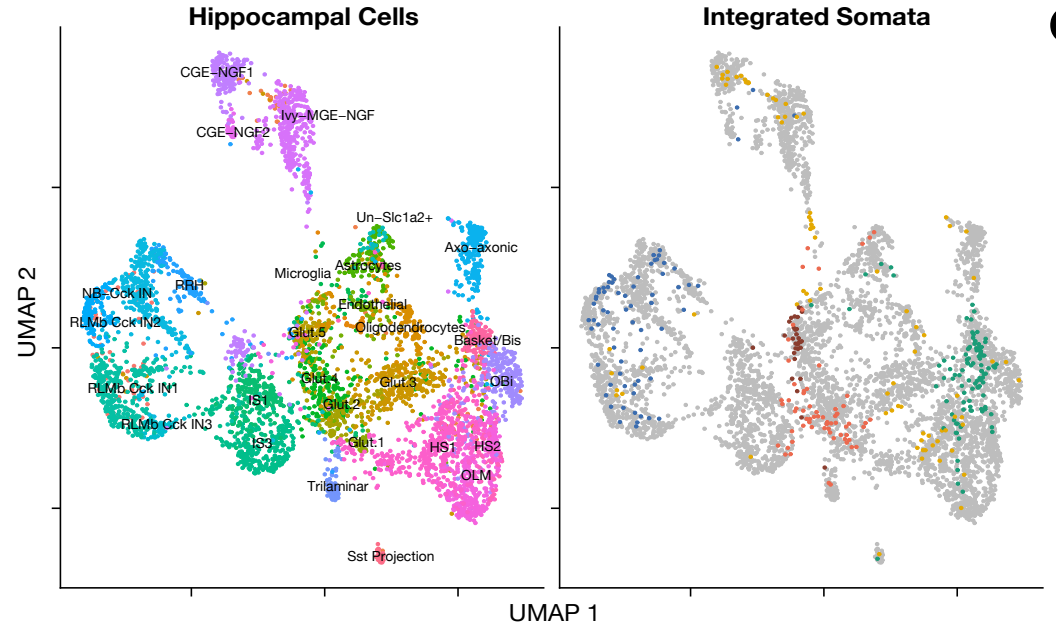

G
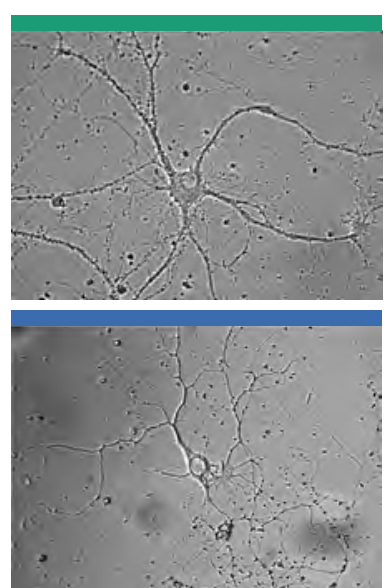
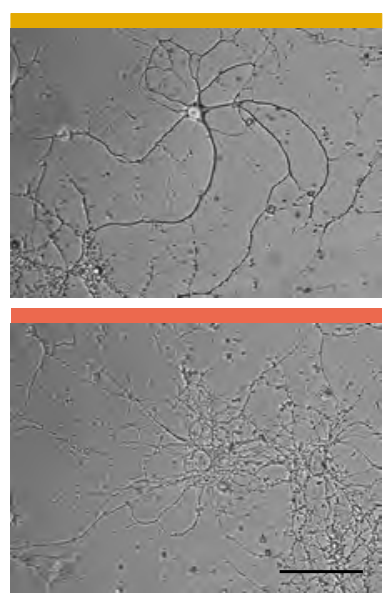
Figure 1. Subcellular scRNA-seq, compartment-specific transcriptomic signatures, and identification of cell types based on the somatic transcriptome. (A) Experimental workflow: After fixation, the soma and dendritic arbor of individual cultured hippocampal neurons were acquired separately by laser capture microdissection. After membrane lysis, mRNAs from a single soma or dendritic arbor were reverse transcribed using a primer containing a primer binding site (PBS), unique molecular identifier (UMI) and a sample index. A template switch reaction added a complementary PBS at the mRNA 5' end. Samples were then pooled, amplified, and prepared for paired-end sequencing. See also methods and Figure S1B for a description of the sequencing data analysis. (B) Number of transcripts detected in different sample groups. (C) UMAP plot of somatic and dendritic samples, colored by cluster identity. (D) UMAP plot of somata samples, colored by cluster identity, revealed two glutamatergic types and three GABAergic cell types. (E) Expression of marker genes projected onto A UMAP plot. (F) Seurat integration was used to find cell type correspondence between hippocampal tissue single neurons (from two separate studies Zeisel et al., 2015 and Harris et al. 2018) and single somata acquired in this study. Left panel shows the cluster organization of hippocampal tissue cells only, and right panel shows the integration of single somata from this study (color indicates cell-type as established in A) with hippocampal tissue cells (gray). For left panel: Basket/Bis: Basket and bistratified cells, CGE-NGF: Caudal ganglionic eminence neurogliaform, Glut: Glutamatergic, HS: Hippocampo-septal interneuron, IS: Interneuron-selective interneuron, IVy-MGE-NGF: Ivy medial ganglionic eminence neurogliaform, $N B$ : Cck+ non-border interneuron, $O B i$ : Oriens-bistratified neurons, OLM: Oriens-lacunosum-moleculare, Sst+ projection neurons, RLMb: Cck+ Radiatum-lacunosum moleculare border interneuron, $R R H$ : Radiatum-Retrohippocampal neurons. (G) Images of representative cells (that were subsequently harvested using laser capture) exhibiting morphology most commonly observed among identified celltypes. Color of bar over image indicates cell-type as indicated in A. Scale bar $=75 \mu \mathrm{m}$. 

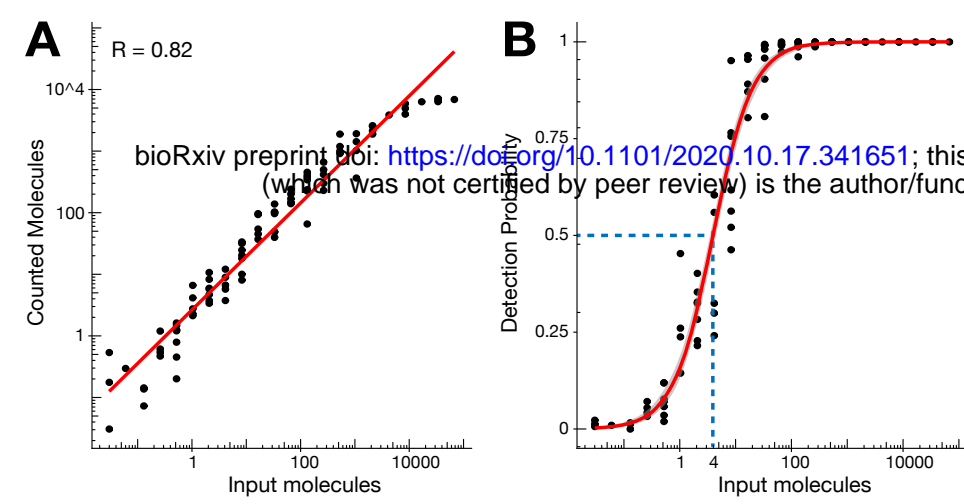

C
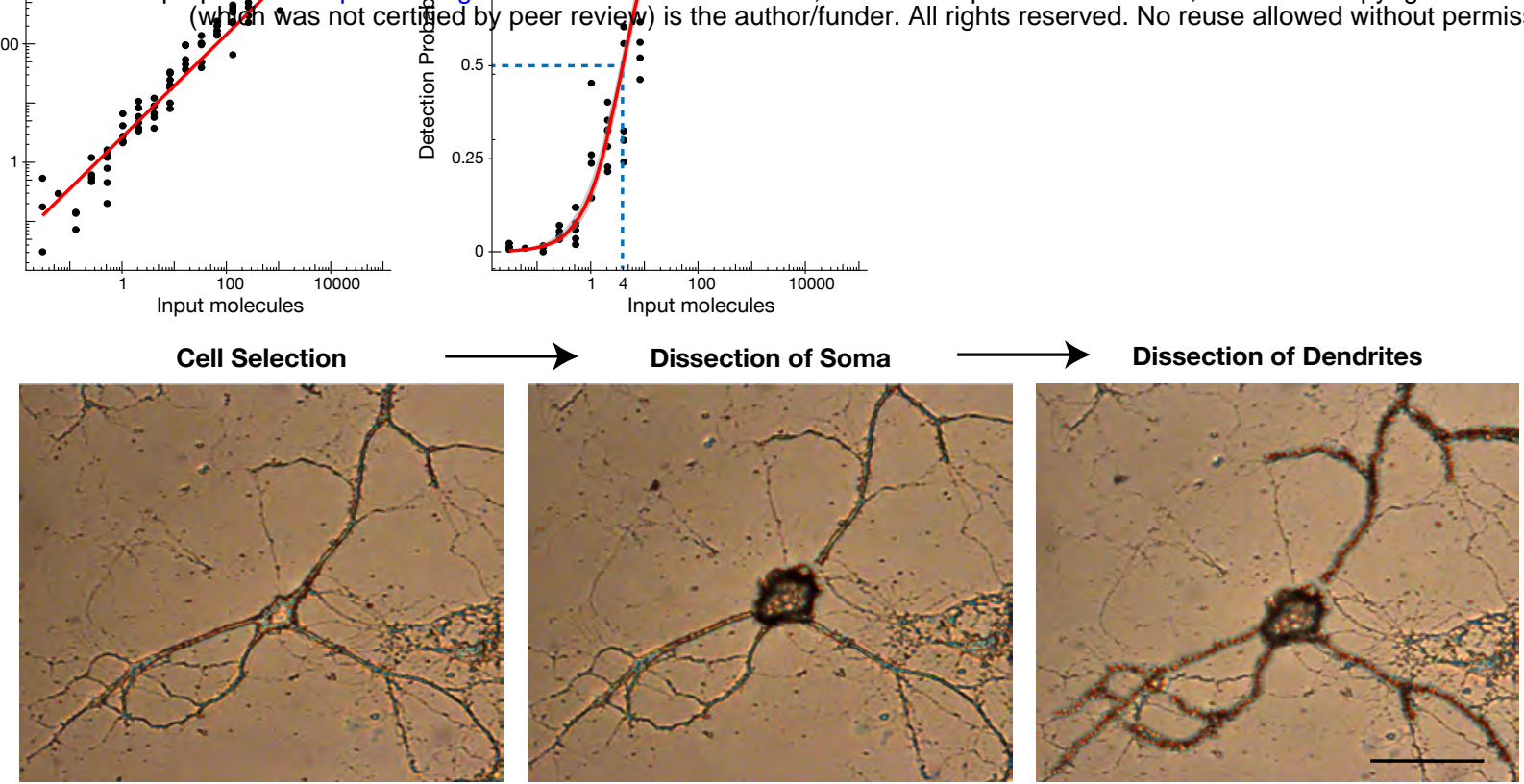

D
Demultiplex
Samples
Extraction \& QC of
(bcl2fastq)
UMI \& Sample Index

Read Quality

Filters

(Drop-seq tools)

$\rightarrow \begin{aligned} & \text { Genome } \\ & \text { Alignment } \\ & \text { (STAR) }\end{aligned}$

$\rightarrow$

Digital Expression

E
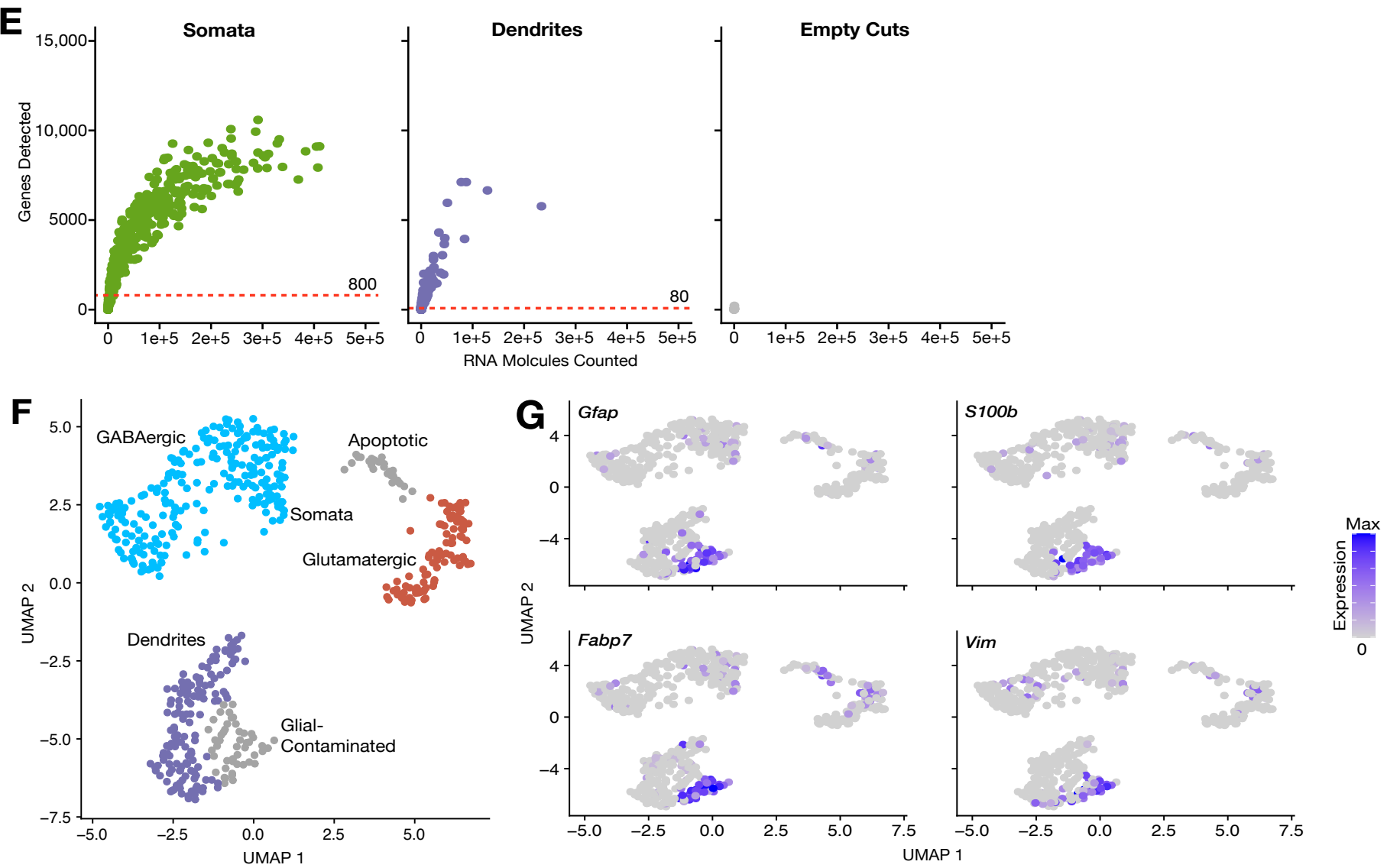

H

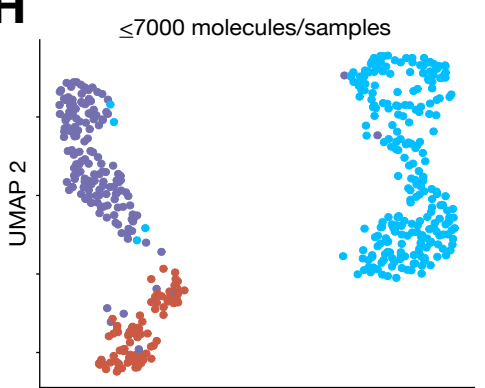

$\leq 1000$ molecules/samples

Downsampling

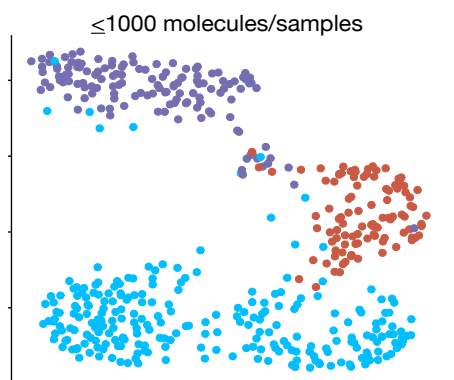

$\leq 4000$ molecules/samples

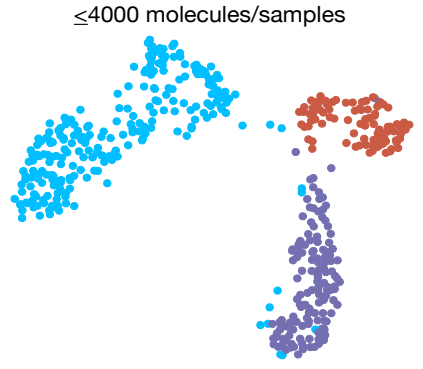

Data

$\leq 500$ molecules/samples

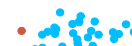

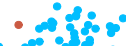

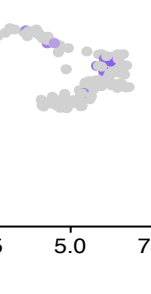



tools)

Analysis

(Seurat) 
Figure S1. Subcellular scRNA-seq, genes detected and compartment-specific transcriptomic signatures. (A) Accuracy of the method is shown by comparing the number of input molecules and average number of detected molecules for each ERCC RNA standard. $R$ indicates Pearson correlation (B) Sensitivity of the method is shown by comparing the number of input molecules for each ERCC and their detection probability (fraction of samples in which the ERCC is detected). Red line shows the mean relation using a fractional logistic regression model. Confidence bands correspond to $95 \%=$ confidence interval. Calculation of detection limit is shown by blue dashed lines indicating the point in which the relation reaches $50 \%$ detection rate when the RNA species contains 4 molecules, suggesting 1 in 4 molecules present are detected. (C) Images showing the dissection of a neuron's soma and dendrites using LCM. Scale bar $=50 \mu \mathrm{m}$. (D) Analysis pipeline of Subcellular scRNA-seq data. (E) Relation between molecules counted and genes detected in each sample group, shows that detection of genes in somata begins to saturate after approximately 100,000 molecules are detected. Saturation is not approached in dendritic samples, likely due to insufficient detection of molecules. Red dashed lines show the gene detection cutoffs used to remove low-content samples. (F) UMAP clustering of all samples sequenced, colored according to the main groups identified. (G) Expression of glial markers among sequenced samples. (H) UMAP clustering of somatic and dendritic samples, after various degrees of randomly downsampling the somatic RNA content. 
clusters expressing either glial or apoptotic markers (Figures S1F-S1G); these were removed from further analyses. After the above filtering steps, 347 somata and 116 dendrites remained.

\section{Somatic and dendritic samples exhibit distinct transcriptomic signatures}

We first investigated the similarities between somatic and dendritic samples. If dendrites simply reflect somatic expression we would expect them to group together with their respective soma in a transcriptome-based clustering analysis. As seen by UMAP visualization, however, cellular samples clearly separated according to subcellular compartments (Figure 1C). Somata were further differentiated between GABAergic and Glutamatergic types, while dendrites remained as one cluster. To determine whether differences in transcriptome depth (Figures $1 \mathrm{~B}$ and $\mathrm{S1E}$ ), were responsible for the observed clustering, we randomly downsampled somata to different molecular totals, comparable to those observed in dendrites. Although the differences between compartments and cell types became less explicit, dendrites still formed a separate cluster while somata continued to split according to cell-type (Figure $\mathrm{S} 1 \mathrm{H}$ ). Together these observations suggest that intercellular diversity is less pronounced in dendrites than in somata, but do not rule out cell-type specific effects in the dendritic transcriptome. Additionally, they imply significant differences exist between the dendritic and somatic transcriptomes of the same neuron.

\section{Identification of cell types based on the somatic transcriptome}

To investigate the effect of cell type on the dendritic transcriptome, we first characterized the cell-types present in our dataset using our somatic samples. Dimensionality reduction among somata revealed five neuron-types: two glutamatergic and three GABAergic (Figure 1D). Figure S2A shows the most differentially expressed genes between identified cell types (adjusted $\mathrm{p}<0.05$, logistic regression model). The major differences in expression occurred between glutamatergic and GABAergic somata, however, significant differences between subtypes were also observed. Although glutamatergic types 1 and 2 largely co-expressed a majority of transcripts, a handful of mRNAs like Nptx2 and Grm5 

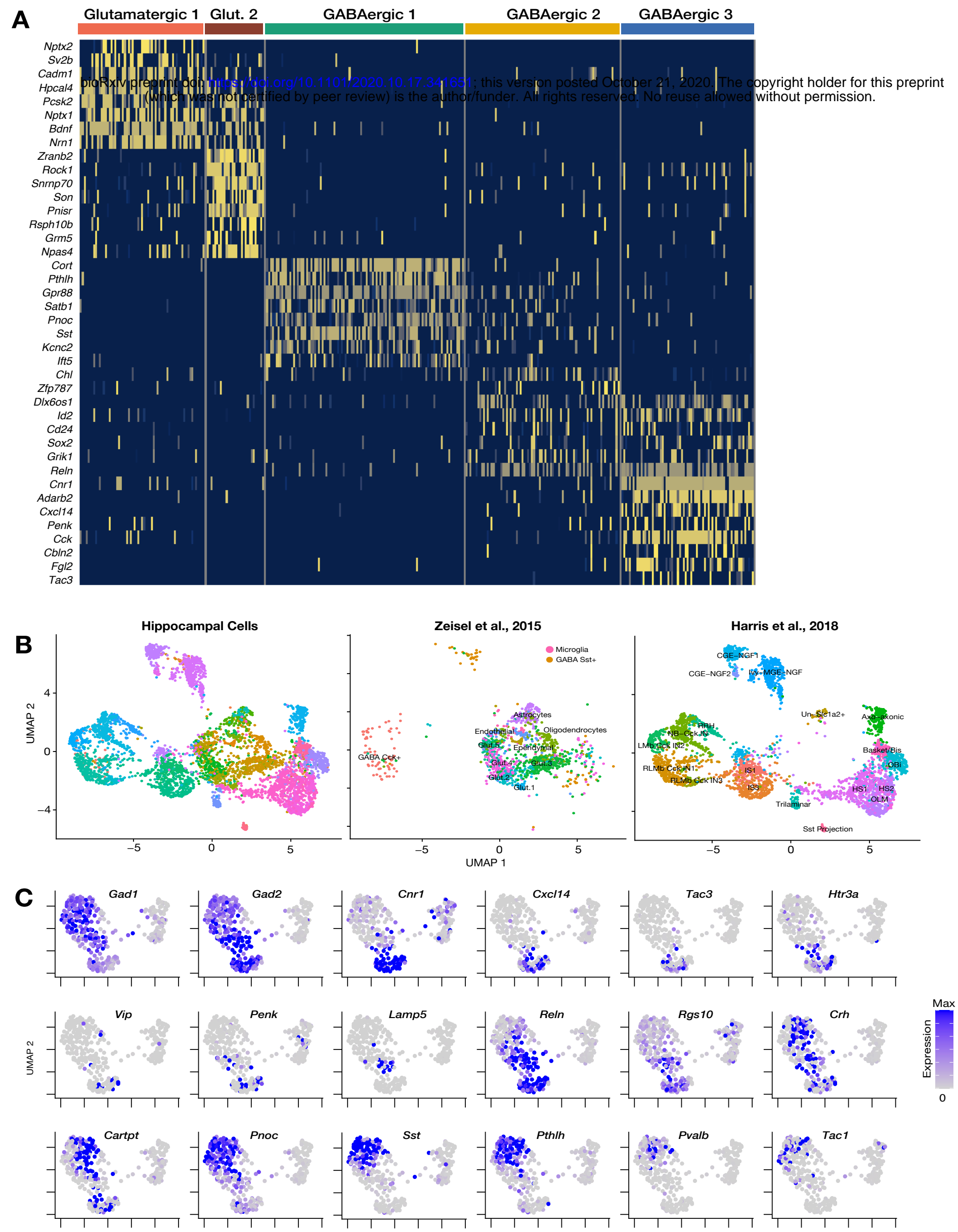

UMAP 1 
Figure S2. Identification of cell types based on the somatic transcriptome

(A) Heatmap showing genes most differentially expressed between cell types. Each column indicates a single soma. (B) Left panel shows Seurat integration of somata dataset and two scRNA-seq datasets derived from the hippocampus. Middle panel shows localization within the plot of cells from Zeisel et al., 2015. Right panel shows localization within the plot of cells from Harris et al., 2018. See Figure 1 legend for a full description of abbreviated terms. (C) Expression of inhibitory interneuron markers projected onto Figure 1D UMAP plot. 
allowed their distinction (Figure 1E). GABAergic 1 neurons, the most abundant cell type in our dataset, were distinguished by their expression of Sst and other neuropeptides. Despite their clear inhibitory identity, GABAergic 3 neurons exhibited a very different transcriptomic profile from GABAergic 1, mainly distinguished by the expression of Cck and Cnr1. Finally, GABAergic 2 neurons showed similarities in their expression patterns to both GABAergic 1 and 3; their identity was best described by the co-expression of some GABAergic 3 markers, like Id2, but not others, like Cck (Figure 1E and S2A).

To benchmark the breadth of cell-types detected we compared our cell-types to those previously described in single-cell studies of the hippocampus. For this we used Seurat.v3 integration algorithm (Butler et al., 2018), which identifies shared populations between different single cell datasets and projects them into a common space. We compared our somata dataset with two CA1-derived scRNA-seq datasets. These studies sampled CA1 cells and profiled the transcriptome of 1314 CA1 cells (Zeisel et al., 2015) or 3,663 CA1 GABAergic neurons (Harris et al., 2018). Our own clustering analysis of this dataset identified 12 or 19 cell types, respectively (data not shown), consistent with the authors' observations. Integration of these two tissue datasets and our somata samples organized cells into multiple glutamatergic, GABAergic and non-neuronal cell types (Figure 1F and S2B). Our glutamatergic 1 somata mainly distributed among hippocampal glutamatergic 1 and 2 neurons, while glutamatergic 2 somata overwhelmingly integrated within the hippocampal glutamatergic 5 cluster. Among the shared marker genes between tissue and cultured cells were Schip1 and Nrgn for glutamatergic 1 somata, and Camk2a and Grm5 for glutamatergic 2 somata. GABAergic 1, 2 and 3 somata showed clear differences in their integration patterns. GABAergic 1 somata were mainly split according to the expression of Pvalb among other genes. Pvalb+ GABA1 somata integrated among Basket-bistratified interneurons, while Pvalb- GABA1 somata integrated among Hippocampo-septal (HS), Oriens-lacunosum-moleculare (OLM) and oriens-bistratified neurons. On the other hand, GABAergic 3 somata integrated exclusively in Cck+ neighborhoods, particularly onto Radiatum-lacunosum moleculare border interneurons (RLMbs). Finally, GABAergic 2 somata were split between different types of 
Neurogliaform and HS neurons. Figure S2C shows the expression patterns of more markers of GABAergic interneurons in our somata dataset.

These transcriptomic relations were paralleled by qualitative similarities in cell morphology. GABAergic 1 neurons tended to have large somata, and large and lengthy dendritic arborizations (Figure 1G). The hippocampal cells they most resemble, Basketbistratified, HS and OLM interneurons, have their somata in the stratum pyramidale (sp) of the hippocampus, and exhibit I complex dendritic arborizations that extend into stratum lacunosum moleculare (slm) (Pelkey et al., 2017). Most GABAergic 2 and 3 neurons had smaller somata, and thin, relatively small dendritic arbors (Figure 1G). Consistently, their transcriptomes were closest to RLMb and Neuroglialform interneurons whose somata are located at the border between the stratum radiatum (sr) and the slm and exhibit short dendrites that usually extend horizontally within layers. Finally, cultured glutamatergic neurons possessed a high density of peri-somatic processes and extended multiple thin proximal dendrites (Figure 1G). Together these results reveal some clear cell-type identities within hippocampal cultures which recapitulate both transcriptome signatures and morphological patterns observed in tissue.

\section{Cell-type specific effects: the dendritic transcriptome of GABAergic interneurons}

Profiles of the neuronal local transcriptome have been derived from populations containing diverse cell types or from populations enriched with Glutamatergic neurons. Instead, our single cell approach allows us to determine the local mRNA pool of cells classified according to their (somatic) transcriptome, including GABAergic types. We detect over 4,000 mRNA species in the dendrites of the glutamatergic and 3 GABAergic neurons (Figure 2A and Table S3). This number is consistent with the number of translated mRNAs recently observed in the hippocampal neuropil (Glock et al., 2020) and represents $\sim 1 / 5$ of the transcripts detected in somata of each cell type, suggesting similar patterns of mRNA localization in glutamatergic and different GABAergic types (Figure 2B). The dendritic transcriptome of GABAergic neurons exhibited both similarities and differences to the glutamatergic dendritic transcriptome (Figure 2A). For instance, Calm1 

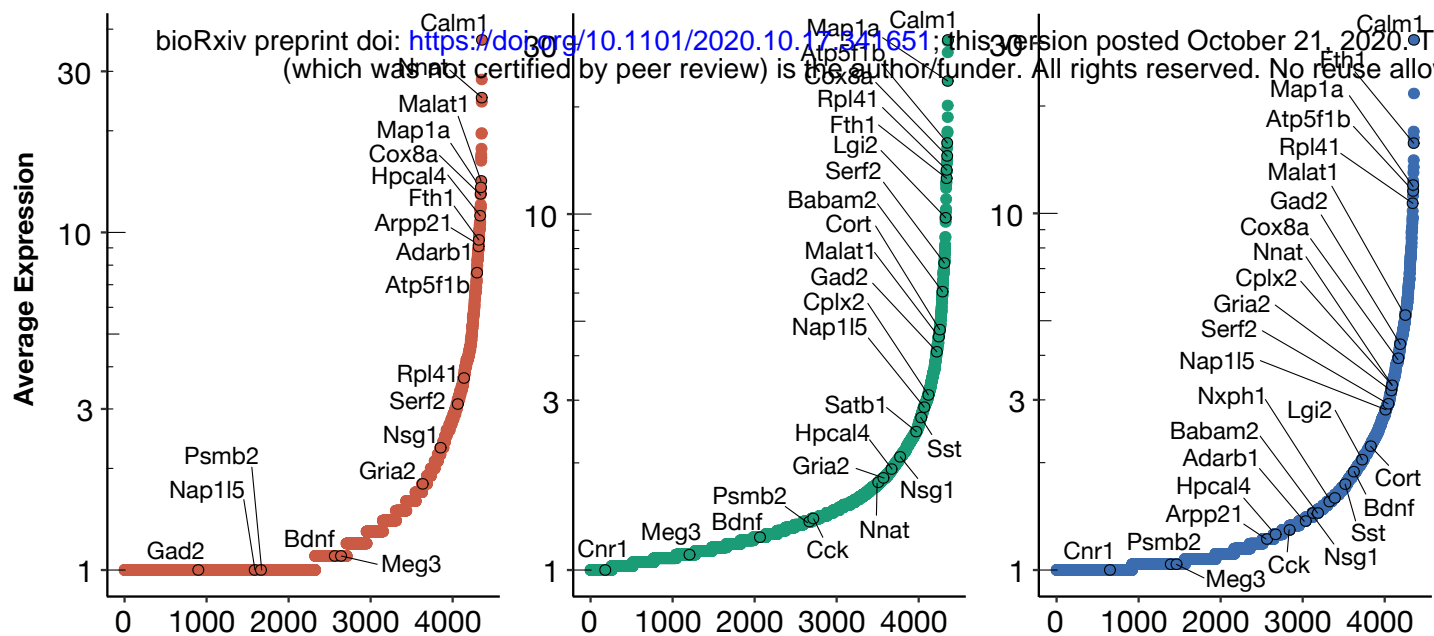

Calm1
AgSint 0

\section{B}

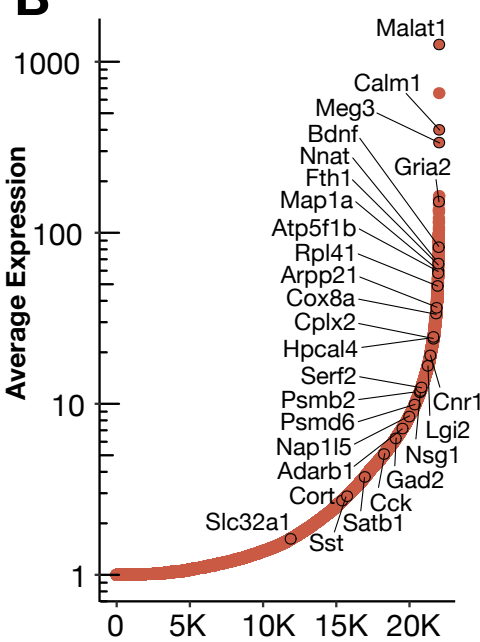

\section{Somata}

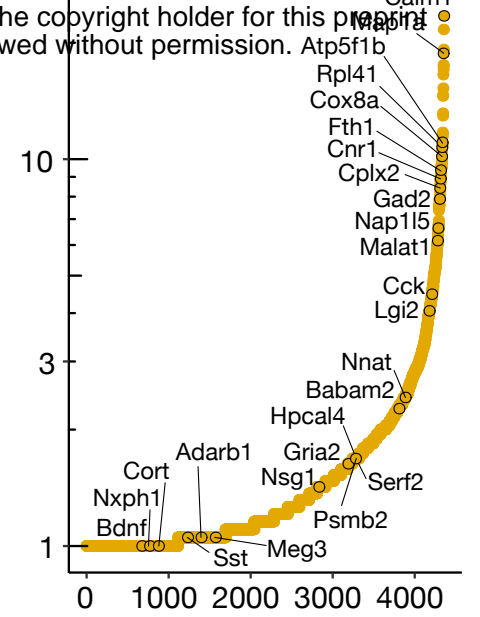

C
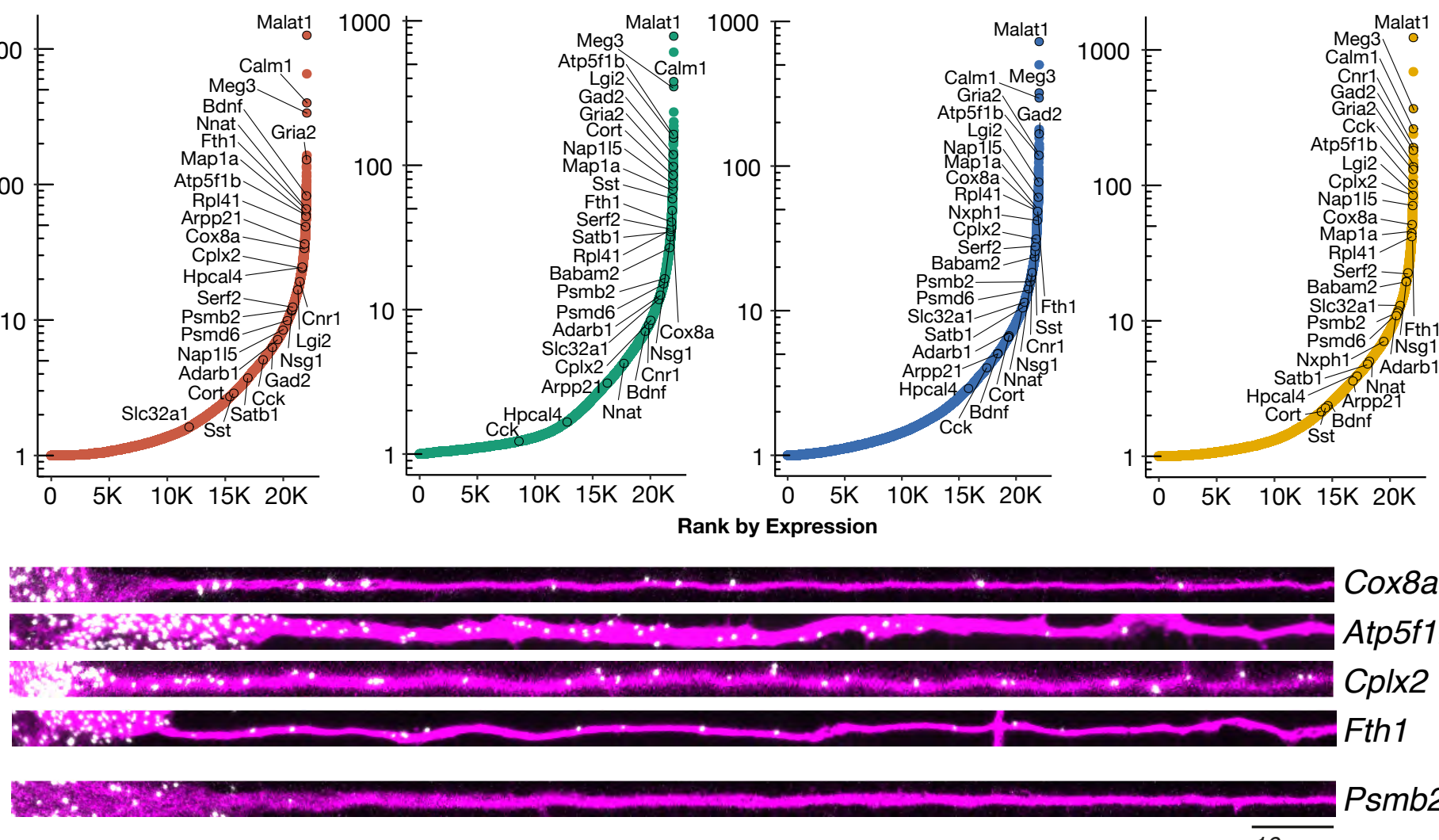

Rank by Expression

Cox8a

Atp5f1b

Cplx2

Fth1

Psmb2 
Figure 2. The dendritic transcriptome of GABAergic interneurons. (A) Plots showing the average expression of genes detected in the dendrites ( $\geq 1$ molecule per sample on average) of the indicated cell types. X-axis shows genes ranked according to their expression from lowest to highest. Some genes are indicated by name. $\mathrm{N}=$ Glutamatergic (10), GABAergic 1 (38), GABAergic 2 (27), GABAergic 3 (19). (B) Same as A but for somatic samples. $N=$ Glutamatergic (95), GABAergic 1 (103), GABAergic 2 (80), GABAergic 3 (69). (C) smFISH for indicated mRNAs observed in GABAergic dendrites (in red). Representative straightened dendrites from Gad-positive neurons extracted from experiments as shown in Figure S3D and oriented from soma (left side) to distal dendrite (right side). Scale bar $=10 \mu \mathrm{m}$. 
and Map1a were among the most abundant dendritic RNA across all cell types. We also observed similarly low abundances in the dendrites of all cell types for genes like Meg3 and Psmb2, which are robustly expressed in the somata of all cell types (Figure 2B). Conversely, we observed mRNAs whose dendritic abundances changed according to cell type including Lgi2 and Serf2, which were most abundant in GABAergic 1 dendrites, and Cnr1 and Cplx2 which were most abundant in GABAergic 3 dendrites. GABAergic 2 neurons exhibited comparable levels of dendritic mRNA species to the other cell types, but did not appear to preferentially localize any mRNA species. To validate the presence of mRNAs in GABAergic dendrites with an independent method, we performed two-color single molecule FISH (smFISH) for selected candidates (Figure 2C) in cells expressing Gad1/2. As predicted from the scRNA-seq data, Cox8a, Atp5f1b, Fth1 and Cplx2 mRNAs were all detected in proximal and distal dendrites, while Psmb2 mRNA was mostly absent from dendrites. These results indicate that GABAergic neurons also localize thousands of mRNAs to their dendrites, resulting in both similarities and differences to the glutamatergic dendritic transcriptome.

To determine statistically significant variability in the dendritic transcriptome according to cell type, we tested differential expression (DE) in dendrites according to cell-type for the following comparisons (Figure S3A): Glutamatergic vs GABAergic, GABAergic 1 vs GABAergic 2-3, and GABAergic 2 vs GABAergic 3. The same comparisons were also made between somata. Consistent with unsupervised clustering results above (Figure 1C), substantially fewer genes exhibited significant DE between dendrites than between somata (Figure 3A and Table S4). Nevertheless, valuable information could be extracted from the differentially-expressed genes. Consistent with the observed transcriptomic distances among cell-types (Figure 1D), more DE genes were detected between Glutamatergic and GABAergic dendrites than in either of the GABAergic comparisons. Interestingly, we observed that in each comparison some genes differentially-expressed in dendrites were also differentially-expressed in somata and vice-versa (Figure 3B and S3B). For example, Hpcal4, Cort and Cnr1 were expressed in a cell-type specific manner in both somata and dendrites. However, not all cell-type-specific genes present in somata 


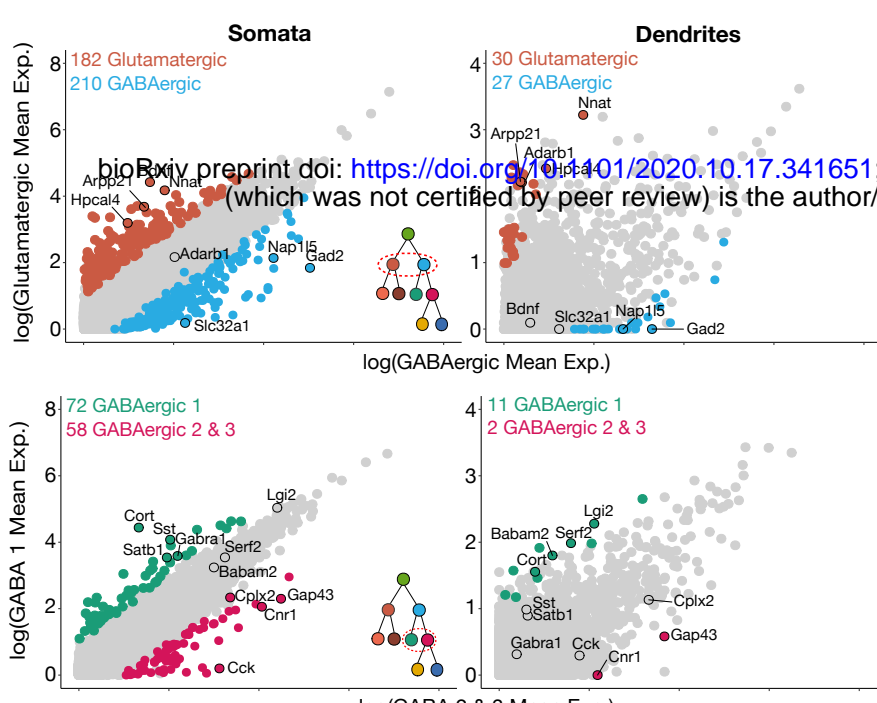

B

(i) Hpcal4 ${ }^{100}{ }^{10}-S_{21}$ Somata Dendrites der. All rights reserved. No reuse allowed witgout permissign.
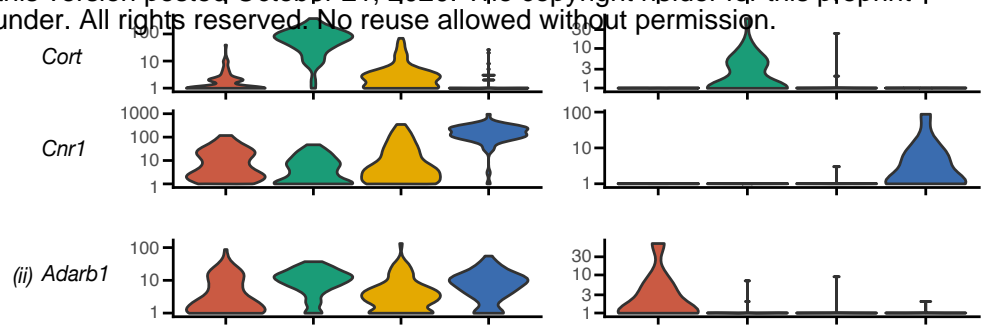

Serf2

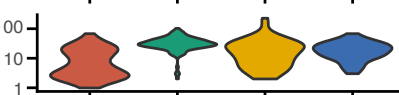

Babam2
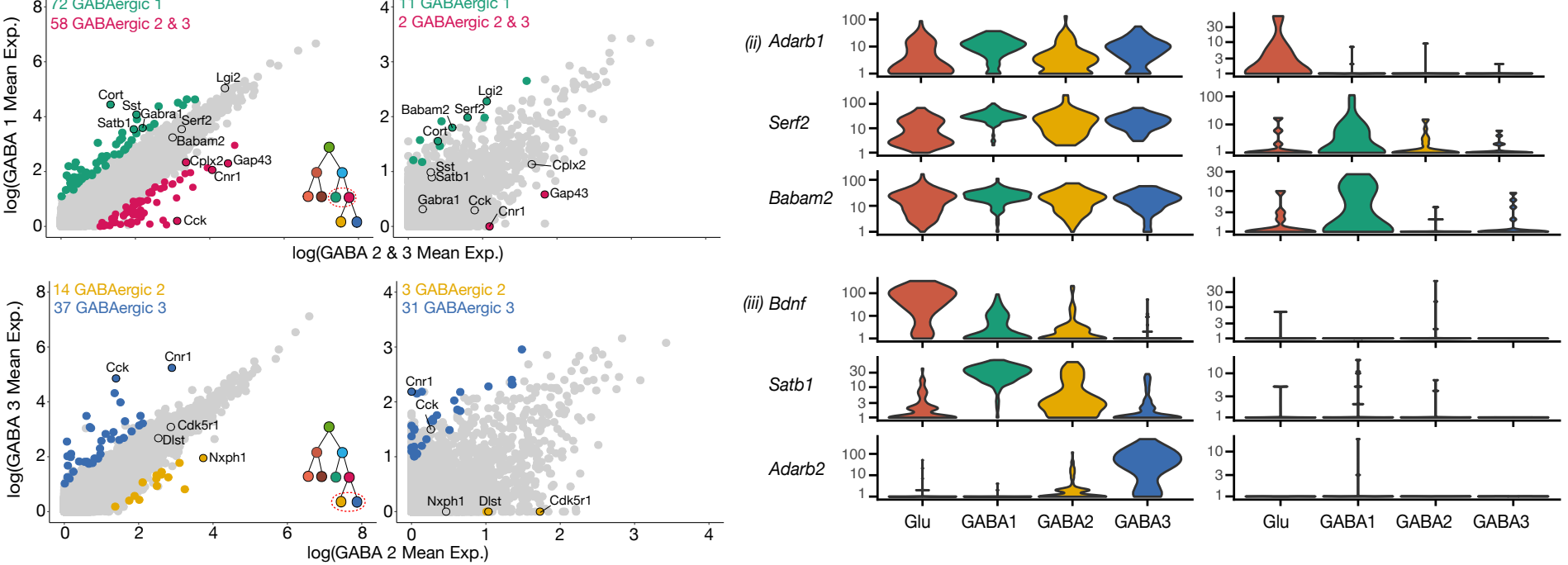

(iii) Bdnf

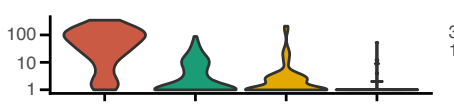

Satb1
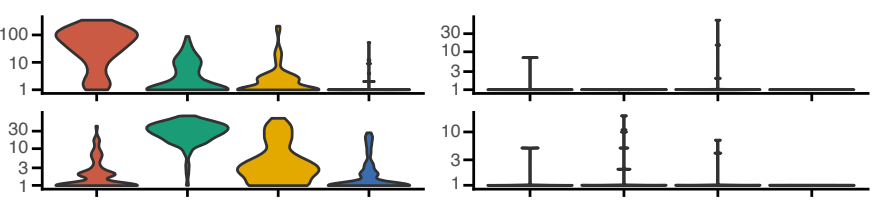

Adarb2
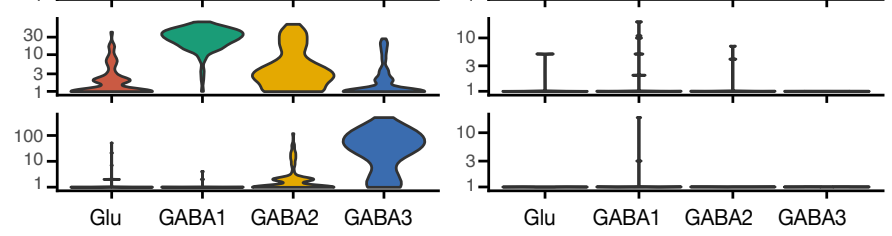

C Hpcal4 Gad1/2

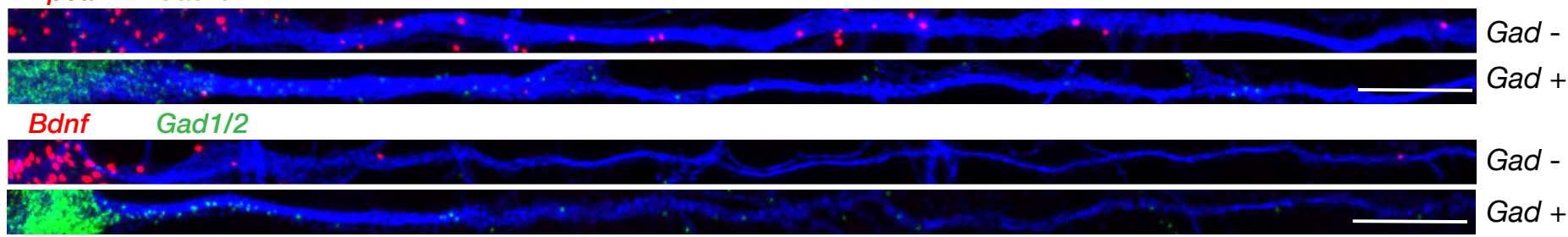

D

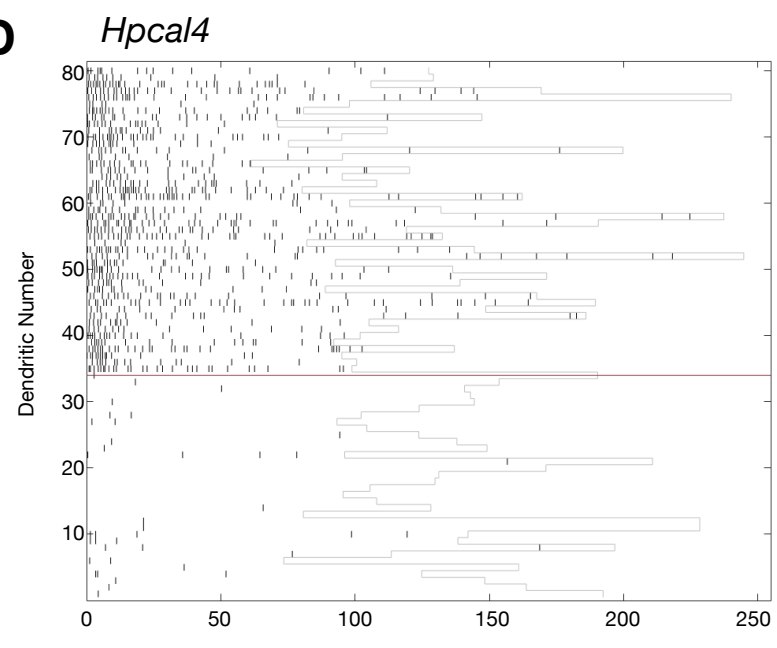

Bdnf

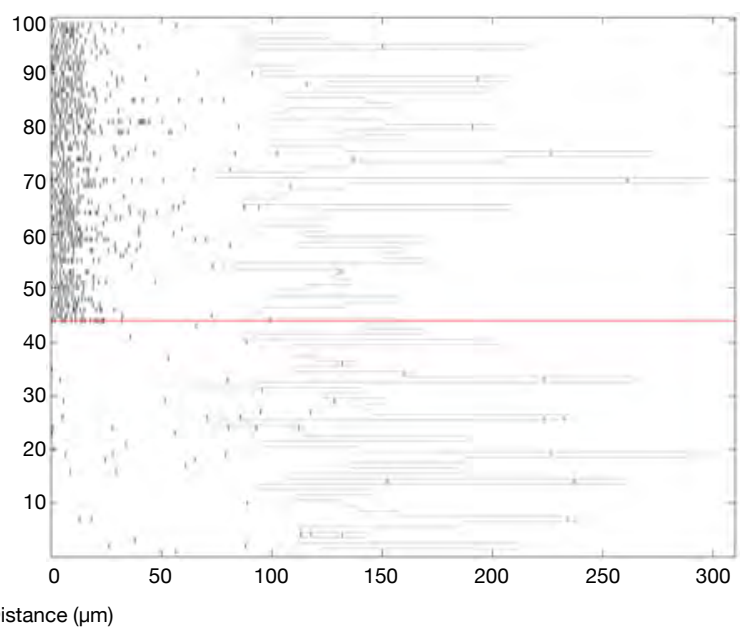

Gad -

Gad + 
Figure 3. Cell-type-specific effects in the dendritic transcriptome. (A) Scatter plots comparing mean expression between somata or dendrites of different cell-types as indicated by $x$ and $y$ axes. Significant differentially-expressed genes ( $p$-value $<0.02$, logistic regression model) are colored in each panel; some significant genes are indicated by name. Insert within scatter plot indicates the comparison been made within the established hierarchy of cell-types (see Figure S2B). Somata N = Glutamatergic (95), GABAergic 1 (103), GABAergic 2 (80), GABAergic 3 (69). Dendrites N = Glutamatergic (10), GABAergic 1 (38), GABAergic 2 (27), GABAergic 3 (19). (B) Examples of cell-typespecific differential expression in (i) both somata and dendrites, (ii) only on dendrites or (iii) only in somata, are shown. (C) Double smFISH for either Hpcal4 or Bdnf (both in red), and Gad1/2 (green). Exemplary dendrites from Gad-negative and -positive neurons extracted from experiments as shown in Figure S3D and oriented from soma (left side) to distal dendrite (right side). Scale bar $=20 \mu \mathrm{m}$ (D) Raster plot showing smFISH dendritic detection pattern over a large number of dendrites from a single representative experiment. Dendrites of both excitatory and inhibitory cells were straightened from the same images, sorted according to the expression of the cell type marker (positive below red line, negative above red line) and the Hpcal4 or Bdnf channels displayed after automated peak detection as raster plot. 
A

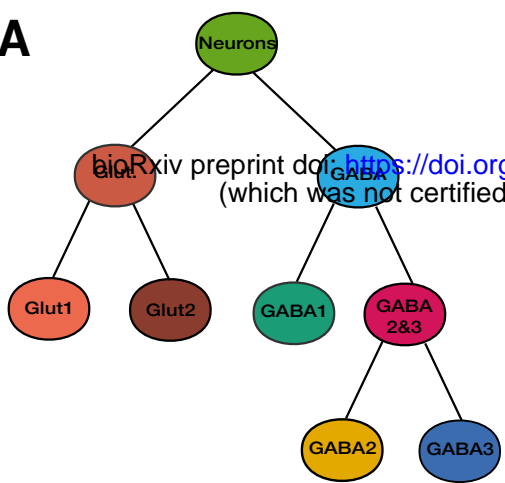

B

Somata

Dendrites

(i)
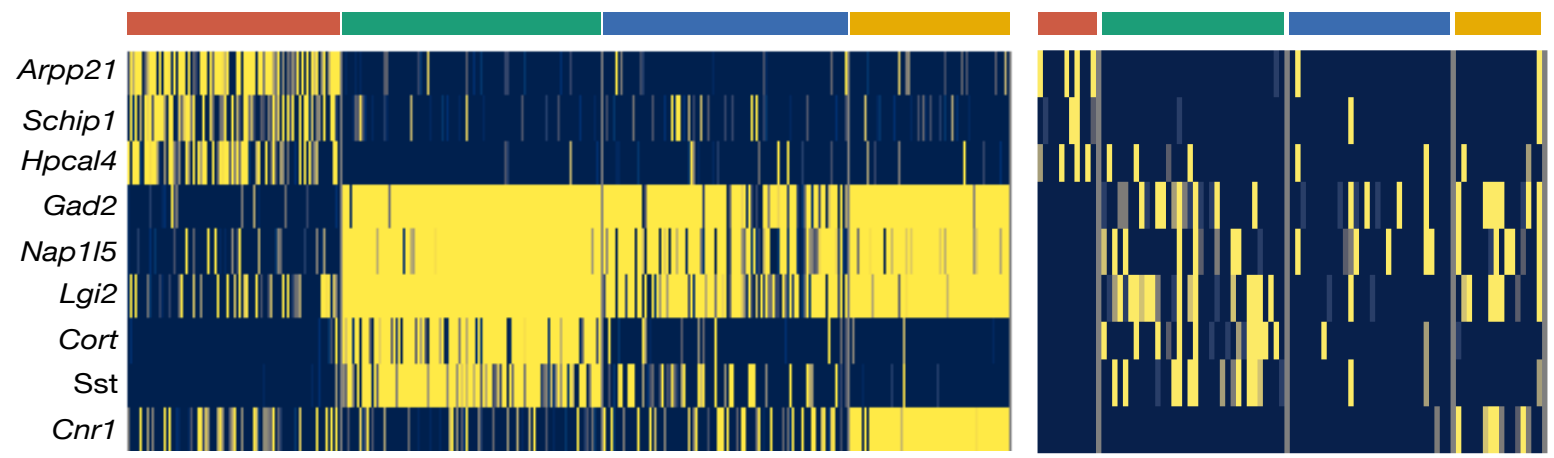

(ii)
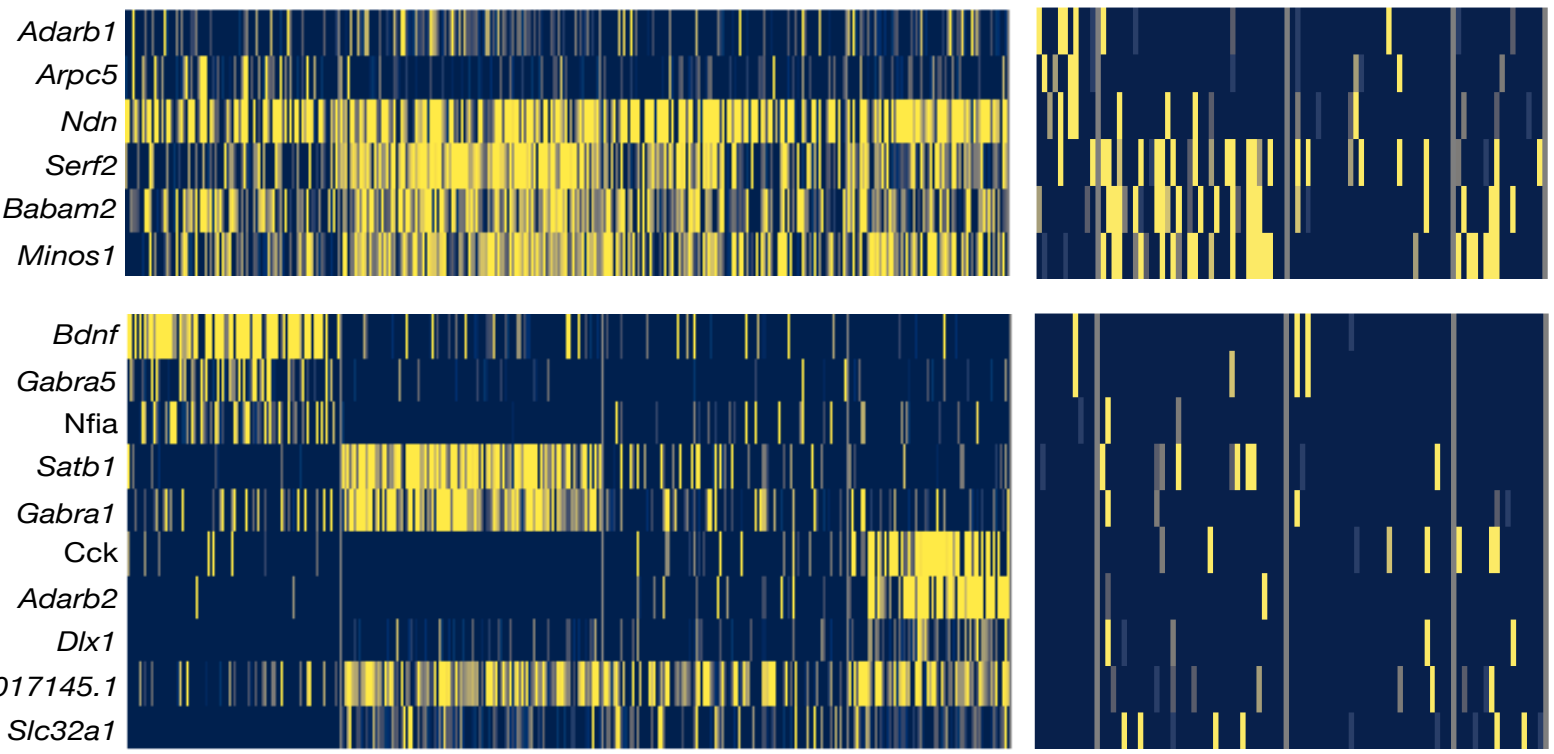

AABR07017145. 1

C
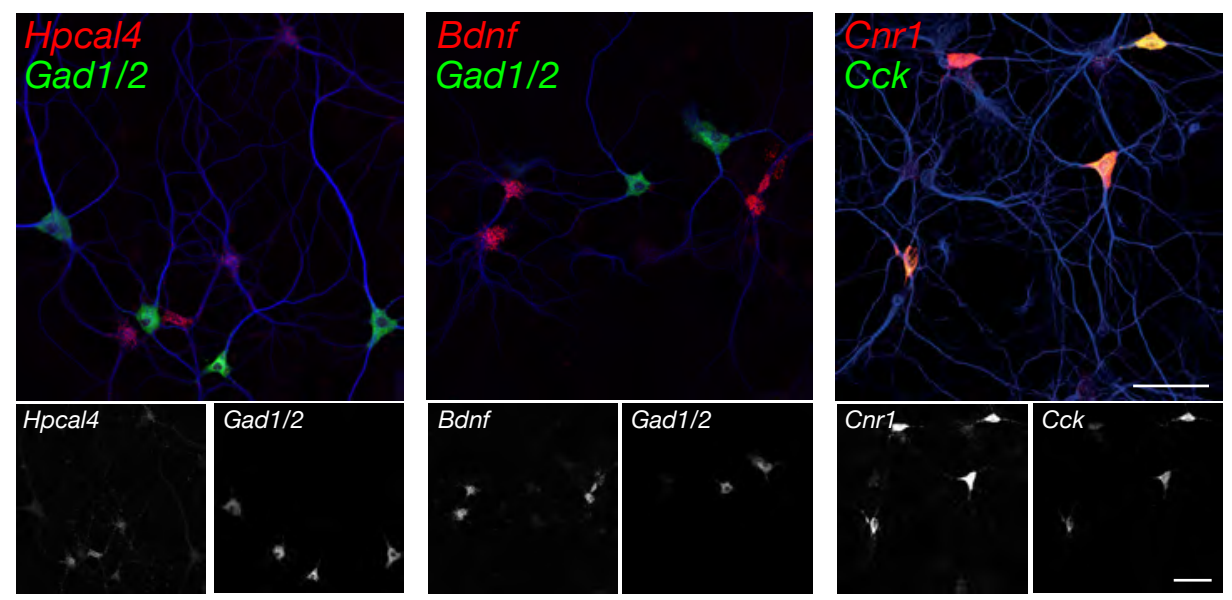

D

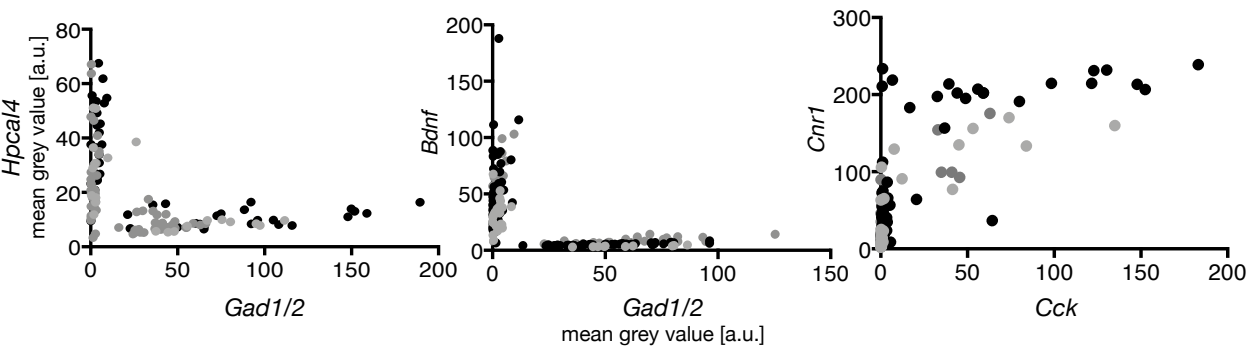

Supplementary

Figure 3 
Figure S3. Cell-type-specific effects in the dendritic transcriptome. (A) Left panel: UMAP plot of dendritic samples, colored by cluster identity. Right panel: same as left panel but colored according to cell-type identity determine by the identity of the respective soma of each dendrite. Orphan dendrites are dendrites whose respective somata did not pass quality controls. (B) Diagram showing the hierarchy of cell types established in clustering analysis (See Figure 2A). (C) Heatmap showing genes differentially expressed between cell types in (i) both somata and dendrites, (ii) only on dendrites or (iii) only in somata. Each column indicates a single soma or dendritic sample. (D) Representative confocal microscopy images of smFISH experiments for Hpcal4 (left), Bdnf (middle) and Cnr1 (right, all in red) and the cell type marker probe sets Gad1/Gad2 (left, middle) or Cck (right, all in green). Neuronal somata and dendrites are shown by MAP2 immunocytochemistry (blue). The two FISH channels are shown separately below each merged image in grey scale (left red; right green). (E) Quantification of FISH intensities in single cell somata of experiments shown in C: both Hpcal4 and Bdnf expression cells separate from the Gad1/2 expressing population. Cells with high Cck expression also express Cnr1. Each dot represents a single cell soma. Different dot colors indicate 3 different experiments. 
were detected in dendrites, e.g. Bdnf, Satb1 and Adarb2. Curiously, we also observed transcripts preferentially detected in the dendrites of specific cell-types even though they were not differentially expressed among somata, suggesting that post-transcriptional mechanisms might act in a cell-type specific manner to regulate mRNA localization. Serf2, Adarb1 and Babam2 are examples of this dendritic pattern.

To validate the above findings with an independent method we performed two-color smFISH for selected candidates, namely Hpcal4, Bdnf and Cnr1 (Figure S3C) together with the cell type marker mRNAs Gad1/2 or Cck. Consistent with the sequencing data, Hpcal4 and Bdnf were expressed in Gad-negative neurons, while high Cnr1 coincided with Cck expression (Figure S3C and S3D). Also, we found that although both Hpcal4 and Bdnf were expressed preferentially in Gad-negative neurons at similar levels, Hpcal4 signal was detected at higher levels in dendrites (Figure 3C), as seen in the sequencing data. To assess variability between neurons we analyzed a larger set of dendrites for these two candidate genes and clustered them with respect to their Gad signal. As expected, Gad-positive cells exhibited almost no signal for either mRNA while Gadnegative neurons clearly showed wide-spread dendritic signal for Hpcal4 but not for Bdnf (Fig. 3D). Together, these data suggest that cell identity can regulate the dendritic mRNA population and that besides cell-type specific transcription, downstream mechanisms may dictate the dendritic localization of specific mRNAs depending on the cell type.

\section{Relation between the somatic and dendritic transcriptomes of single neurons}

It is unknown how much the somatic abundance of an mRNA influences its subsequent localization or not to the dendrites. To investigate this, we focused on 94 neurons for which both soma and corresponding dendrites passed QC filters. We observed that, like the somatic transcriptome, dendritic mRNA abundances roughly resembled a log-normal distribution albeit more zero-shifted (Figure S4A). This indicated that the majority of dendritic mRNAs are present at relatively low levels while a few genes are found at moderate-to-high levels. Using a logistic regression model, we observed that somatic expression significantly influences dendritic detection (Figure $4 A, p=1.29 \times 10^{-6}$ ), but 


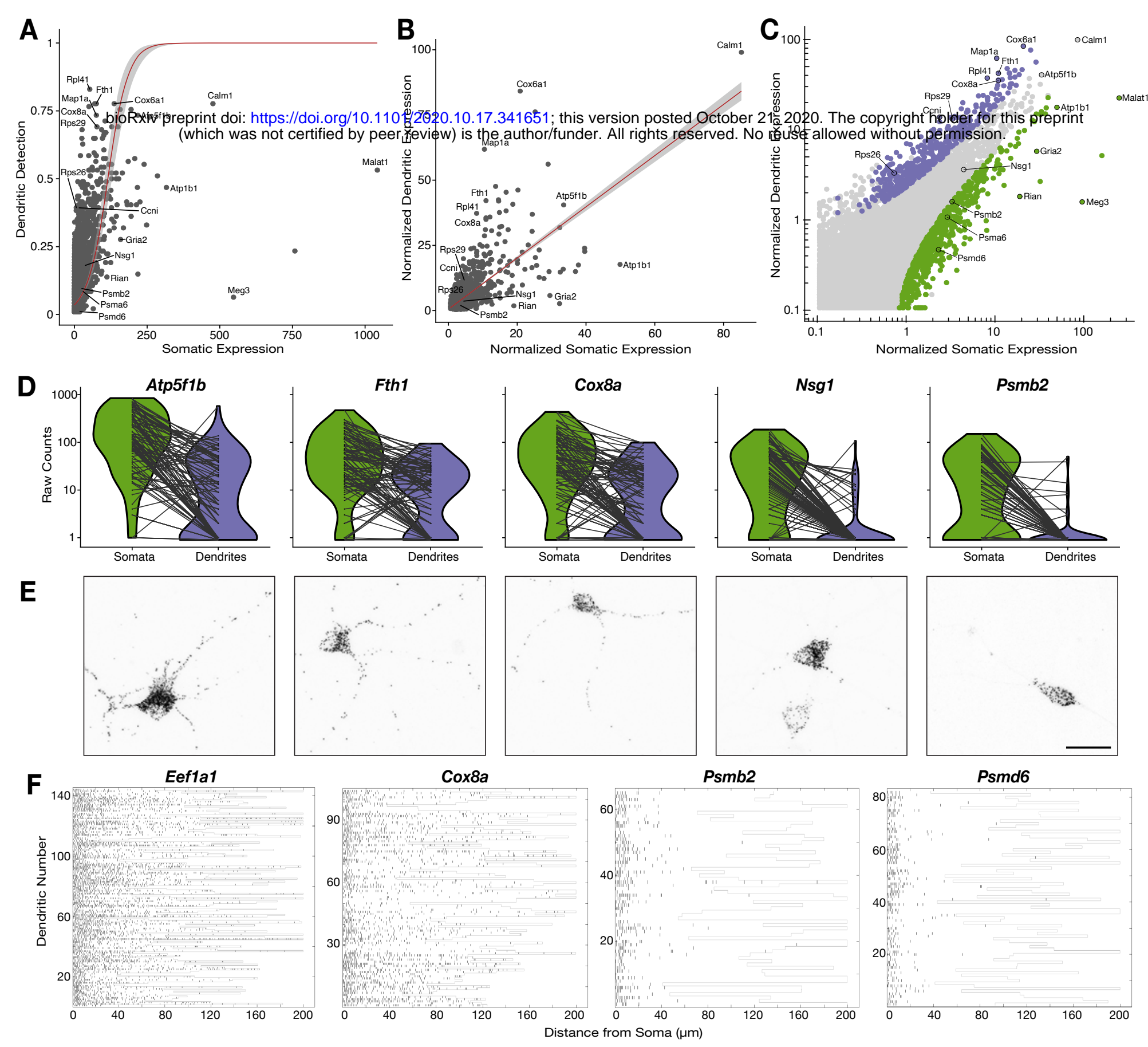


Figure 4. Relationship between the somatic and dendritic transcriptomes of single neurons. (A) Scatter plot showing the relationship between somatic abundance and the fraction of samples in which an mRNA species is detected in the dendrites. A logistic regression model revealed a significant correlation $\left(p=1.29 \times 10^{-6}\right.$, McFadden's Pseudo $\left.R^{2}=0.33\right)$. Gray area indicates the $95 \%$ confidence interval. Some genes discussed in the text are indicated by name. (B) Correlation between somatic and dendritic expression for genes expressed in at least $20 \%$ of dendritic samples. Red curve shows a linear regression model $\left(p=2 \times 10^{-16}, R^{2}=0.50\right)$. Some genes discussed in the text are indicated by name. Gray area indicates the $95 \%$ confidence interval. (C) Scatter plot showing the results of paired differential expression analysis using a Poisson generalized linear model. Colored dots indicate statistically significant genes (adjusted $p<0.05$ ) in somata (green) or dendrites (purple); some significant genes are indicated by name. (D) Violin plots showing the raw counts in somata and dendrites of gene examples. Lines between violins indicate the paired values of soma and dendrites from the same neuron. (E) FISH validations for genes indicated in D. The abundance and distribution of mRNAs detected (black) is shown. Neurons were identified and outlined by Map2 immunochemistry (see images in Figure S4F). Scale bar $=25 \mu \mathrm{m}$. (F) Raster plot showing the cell-to cell variability of mRNA localization as determined by $\mathrm{smFISH}$, for candidate dendritically-enriched (Eef1a1, Cox8a) and dendritically-de-enriched (Psmb2, Psmd6) mRNAs. One dendrite was followed per cell, straightened, and the profile converted via peak detection into a raster plot (displayed from soma/proximal dendrite to distal dendrite, from left to right). 

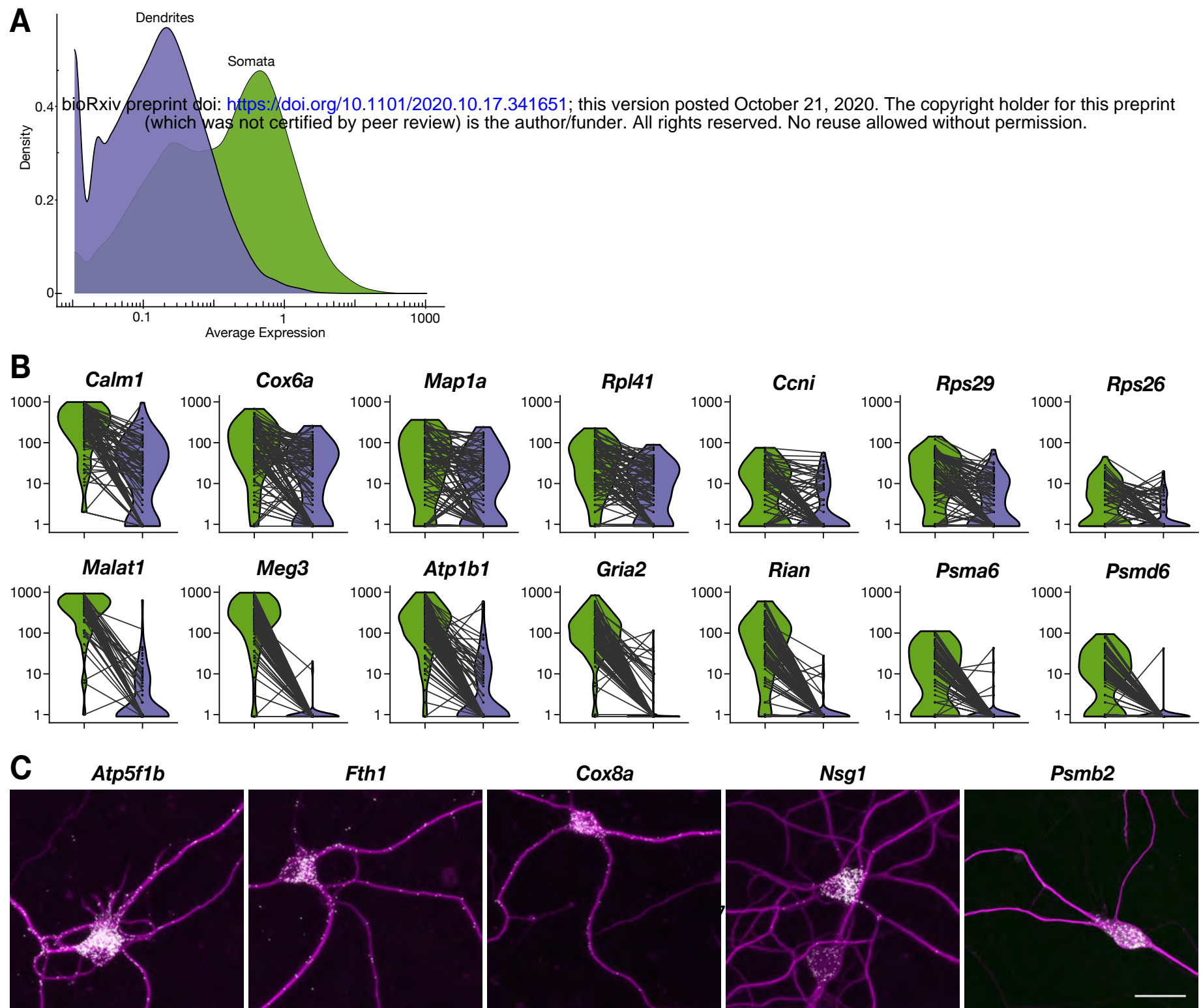
Figure S4. Relationship between the somatic and dendritic transcriptomes of single neurons. (A) Density plot showing the distribution of expression values (logarithmic scale) of dendrites and somata. (B) Violin plots showing the raw counts in somata and dendrites of various genes. Lines between violins indicate the paired values of soma and dendrites from the same neuron. (C) FISH validations for genes indicated in Figure 4D showing the abundance and distribution of mRNAs (white) as shown in Figure 4E, and the neuron structured outlined by Map2 immunochemistry (magenta). Scale bar $=25 \mu \mathrm{m}$. 
only partially explains the observed variance (McFadden Pseudo $R^{2}=0.33$ ). To compare the somatic and dendritic transcriptome we used normalized expression values that stabilize technical variability among samples, for genes present in more than $25 \%$ of samples (Hafemeister and Satija, 2019). Somatic and dendritic expressions were significantly correlated (Figure 4B, generalized linear regression model, $p=2 \times 10^{-16}$ ) but, again, only partially accounted for the observed variance $\left(R^{2}=0.50\right)$. These results indicate that the more abundant an mRNA is in the soma the more likely it is to be present and abundant in dendrites, but additional mechanisms must act to increase or decrease dendritic presence of certain mRNAs.

To identify genes with significant enrichment or de-enrichment in dendrites we performed a paired-differential expression analysis using a Poisson generalized linear model (Stuart et al., 2019), based on the difference between somatic and dendritic values in each single neuron. We used normalized values that correct for the large differences in detected molecules between somatic and dendritic samples (Figure 1B), and thus, allow us to test for relative enrichment (Hafemeister and Satija, 2019). We detected 463 and 764 genes enriched or de-enriched in dendrites, respectively (Figure 4C and Table S5), including most of the genes deviating from the general trends in Figures $4 \mathrm{~A}$ and $4 \mathrm{~B}$. Figure $4 \mathrm{D}$ and Supplementary figure $4 \mathrm{~B}$ shows transcripts in their raw instead of normalized counts, and with compartments paired according to cell, to more accurately present the quantitative nature and variability of individual neurons.

Beside relative enrichment, localization of an mRNA in dendrites may be evaluated based on its absolute local abundance. Indeed, we observed significant enrichment in some but not all genes with high abundance in dendrites. For instance, the mitochondrial genes Atp5f1b, Fth1 and Cox8a, all had high absolute values in dendrites, but only the latter two were significantly enriched (Figure 4D). This is because a lower fraction of the cell's total Atp5f1b mRNA is localized to dendrites in comparison to Fth1 and Cox8a. We also observed significant dendritic enrichments across a wide spectrum of absolute values. For example, the mRNAs of ribosomal proteins Rps26, Rps29 and Rp/41 were all 
comparably enriched in dendrites although they had very different dendritic expression values (Figure S4B). We also observed genes de-enriched in dendrites across a widespectrum of somatic abundances. For instance, Atp1b1, Malat1 and Gria2 were all deenriched in dendrites even though they had moderate-to-high abundances in both dendrites and somata (Figure S4B). In contrast, genes like Meg3, Rian, Psmb2 and Psmd6 were similarly de-enriched and scarce in dendrites, although they are expressed at very different levels (Figure S4B). Since Malat1, Meg3 and Rian are nuclear non-coding RNAs their dendritic de-enrichment is to be expected. However, the severe dendritic deenrichment of genes such as Gria2, Psmb2 and Psmd6 suggests specific mRNAs may be actively deterred from entering dendrites.

To validate these observations, we performed smFISH on five representative genes (Figure 4E and S4C). Consistent with our scRNA-seq dataset, Atp5f1b, Fth1 and Cox8a exhibited similar high abundances throughout the length of dendrites. However, as predicted, Atp5f1b was more abundant in the soma than Fth1 and Cox8a. For Nsg1, a gene that was neither significantly enriched nor significantly de-enriched in dendritic samples, the great majority of the signal was in the soma, with some signal detected in the proximal dendrites. Finally, as seen in our scRNA-seq data, signal for Psmb2 was almost exclusively present in the soma. To characterize the cell-to-cell variability of such patterns we used smFISH to quantify dendritic localization of two dendritically-enriched genes, Eef1a1 and Cox8a, and two dendritically-de-enriched genes, Psmb2 and Psmd6, across hundreds of neurons (see methods; Figure 4F). Both Eef1a1 and Cox8a were highly abundant often reaching far into the dendrites of most neurons. In contrast, Psmb2 and Psmd6 displayed a rapid decay of signal density within the first $20 \mu \mathrm{m}$ of the dendrite. Nevertheless, we observed some cell-to-cell variability in the absolute number of dendritic mRNAs of a gene, as seen in our scRNA-seq data (Figure 4D and S4B). For instance, a handful of dendrites exhibited low expression for Cox8a, while for Psmb2 and Psmd6 a few neurons showed sparse mRNA puncta along the dendrites. Thus, as observed by scRNA-seq data, the smFISH experiments showed different genes substantially differ in 
their dendritic localization and abundance, with some cell to cell variability not accounted by cell-type.

\section{Functional associations of dendritically enriched and de-enriched genes}

To investigate whether mRNA distribution between soma and dendrites is related to function, we performed rank-based gene set enrichment analysis. Genes were ranked by a "representation score", which is the product of their mean expression $\mathrm{x}$ detection frequency, and tested for enrichment in gene ontology cellular and synaptic components (GOCC and SynGO; Koopmans et al., 2019) terms. Most terms were strongly represented in both somata and dendrites, and included essential dendritic functions like energy and mitochondria, cytoskeleton, and synaptic organization and maintenance (Figure 5A and Table S6). Although their local function is still unclear, ribosomal protein mRNAs were also highly represented in dendrites (Cajigas et al., 2012; Glock et al., 2020; Gumy et al., 2011; Poon et al., 2006; Zhong et al., 2006). Interestingly, we also found a set of genes significantly enriched only in dendrites relating to autophagy, calcium channels, and heterotrimeric G-protein complexes. As predicted by Figure 4, we also found a set of terms de-enriched in dendrites including chromosome regulation, splicing, and Golgi-related terms, whose proteins are known to be excluded/diminished in dendrites (Hanus et al., 2016). Intriguingly, proteasome-related terms are also deenriched in the dendritic transcriptome even though, proteasomal proteins are present and play essential roles in dendrites (Bingol and Schuman, 2006). Thus, the local transcriptome supplies dendrites with some but not all of the proteins needed on-site.

We next determined whether the mRNAs encoding proteins that are integral members of macromolecular complexes exhibited similar or differential enrichment in the two compartments. We selected GOCC terms describing macromolecular complexes and analyzed the relative enrichment distribution of the mRNAs in the somata vs. dendrites. Figure 5B shows the distribution of 6 contrasting complexes. The distributions of both heterotrimeric G-protein complexes and ribosomal proteins exhibited above-average dendritic enrichment (Figure 5B and 5C), but the variance was higher in the latter. Both 


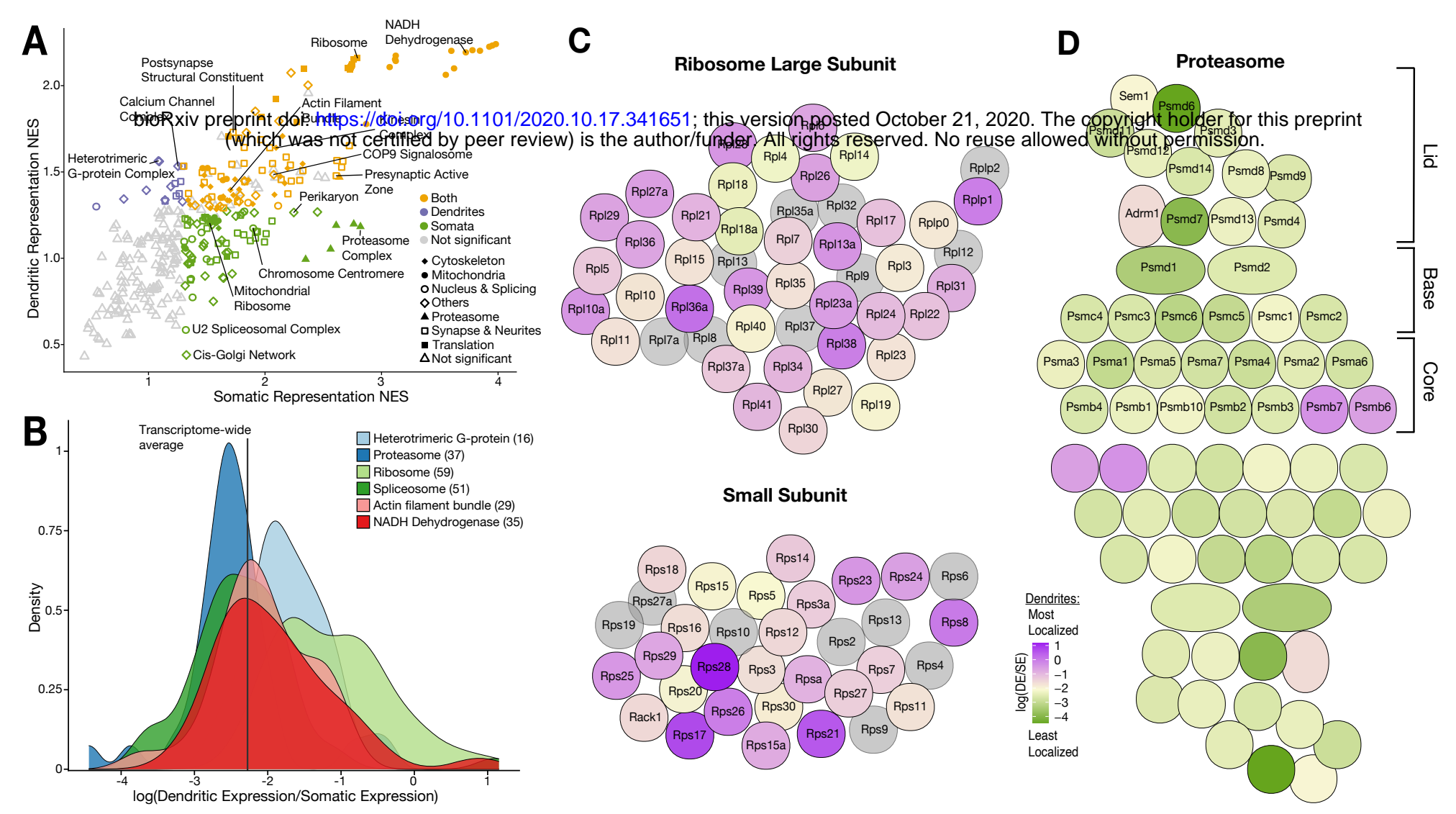


Figure 5. Functional associations of dendritically enriched and de-enriched transcripts. (A) Gene ontology analyses of dendritic enrichments. Shown are the normalized enrichments scores (NES) of different cellular component and synaptic terms (GOCC and SynGO) calculated from ranked-lists of dendritic and somatic mRNAs. Genes were ranked according to a "representation score" which equals detection frequency $x$ mean expression. Terms with a significant enrichment score $(F D R<0.25)$ are colored according to the compartment they were significant in. Some terms are indicated by name. (B) Density plot showing the distribution of the relative dendritic enrichments for genes belonging to six GOCC terms. Relative enrichments equated the average ratio between dendritic and somatic expression in a single neuron. Distributions are colored according to complex and the number of genes evaluated for each complex is shown in parenthesis next to the term in the figure legend $(\mathbf{C})$ Cartoon showing the proteins of the ribosome colored according to the relative dendritic enrichments of their mRNAs. Gray color indicates the mRNA species was not detected. (D) Same as C but for proteins of the proteasome. 
the proteasome and spliceosome were skewed towards below-average dendritic enrichment (Figure 5B and 5D), with the proteasome exhibiting lower variance. These observations suggest that protein function influences mRNA localization patterns.

\section{Local protein synthesis occurs in the dendrites of GABAergic neurons}

As described above, we found that GABAergic neurons localize thousands of mRNAs to their dendrites (Figure 2A), including both cell type variable and invariable transcripts, suggesting these neurons also have the capacity to locally synthesize proteins. To test this directly, we took advantage of a conditional mouse line that enables the expression of a mutant Methionyl tRNA synthetase (MetRS*) in specific cell-types, when crossed to different Cre driver lines (Alvarez-Castelao et al., 2017). Coupled with the administration of the non-canonical amino acid ANL (azido norleucine), protein synthesis was measured in glutamatergic neurons (Nex-Cre::GFP-2A-MetRS*), the GABAergic neurons (Gad2Cre::GFP-2A-MetRS* ${ }^{*}$ and somatostatin-expressing neurons (Sst-Cre::GFP-2AMetRS*). We cultured cortical neurons from these mouse lines on membranes that enabled the separation of neurites from soma-containing fractions (Alvarez-Castelao et al., 2020; Poon et al., 2006), and examined protein synthesis in intact neurons ("full labelling"- ANL added to entire dish) or neurites alone ("neurite only", ANL added after somata layer was removed) using BONCAT (Alvarez-Castelao et al., 2019; Dieterich et al., 2007) (Figure 6A). As expected, full labeling resulted in signal both in the soma and the neurites (Figure 6B and 6C). Following "neurite only" labeling, strong signal was detected in the neurites of all cell types examined, including the GABAergic neurons (Figure 6B and 6C). These results confirm that GABAergic neuron dendrites locally synthesize proteins and suggest this is a pan-neuronal property.

\section{Discussion}

We established a method to characterize the transcriptomes of subcellular compartments in individual neurons which we used to measure both the intercellular variability and intracellular allocation of the dendritic transcriptome. This technique allows for the clean dissection of the dendrites and respective soma from a cell of choice, while preserving 


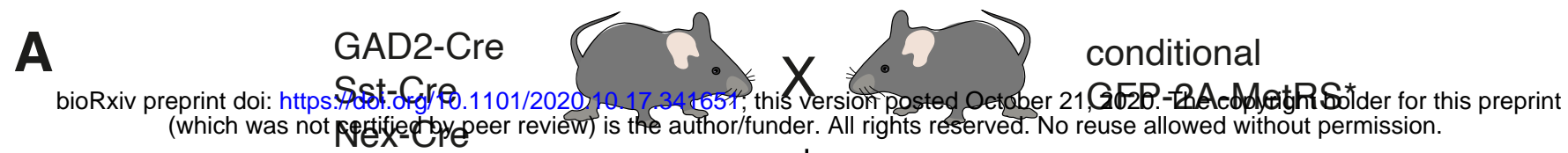

Cre + MetRS $^{*}+$
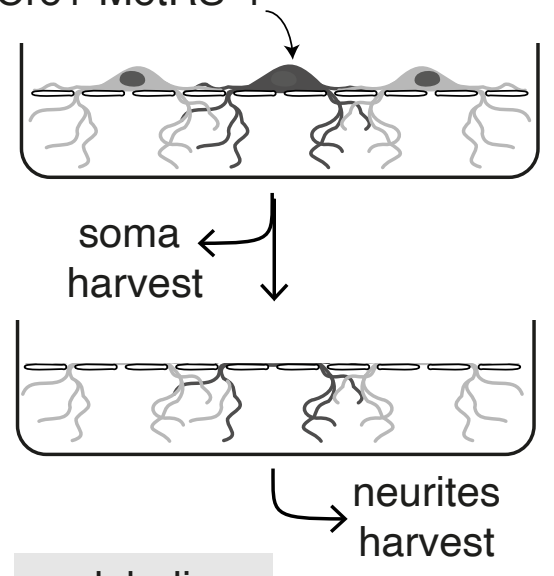

no labeling
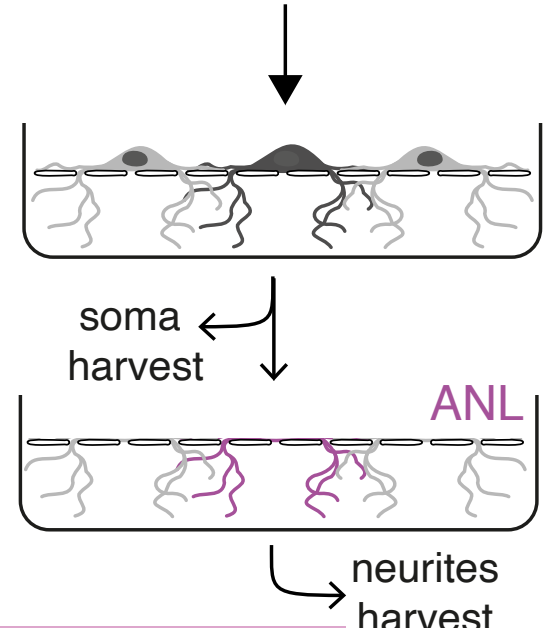

neurite-only labeling
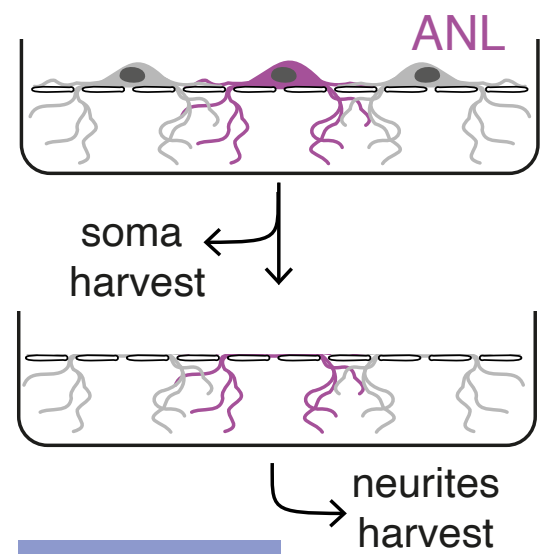

full labeling
B

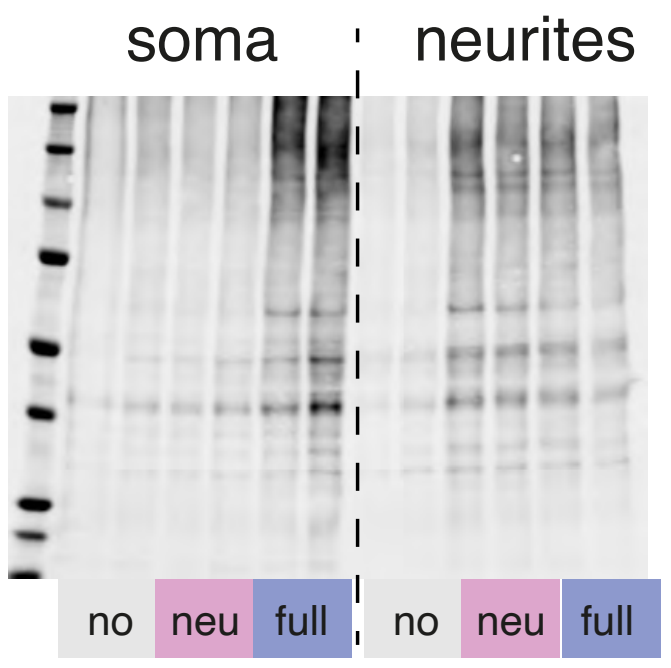

D

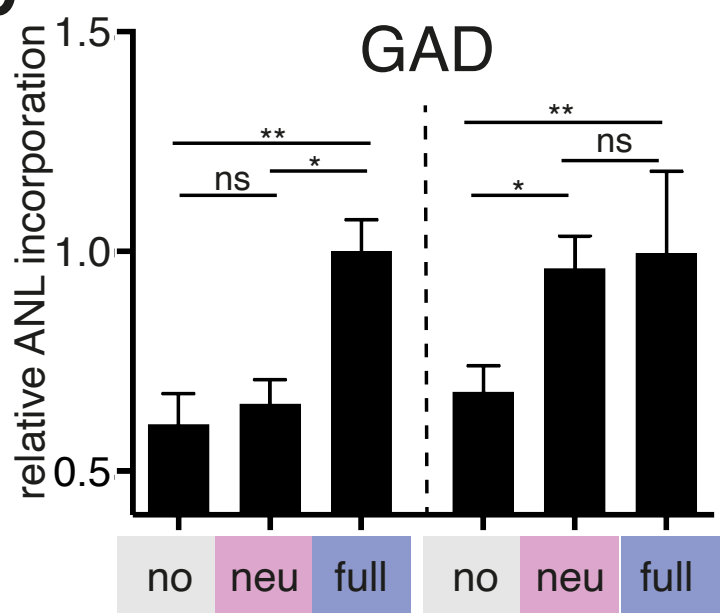

C soma neurites

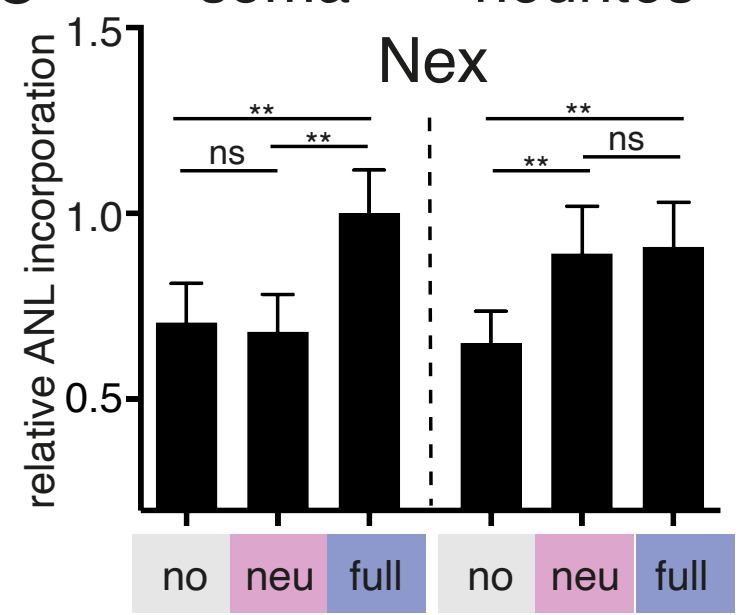

E

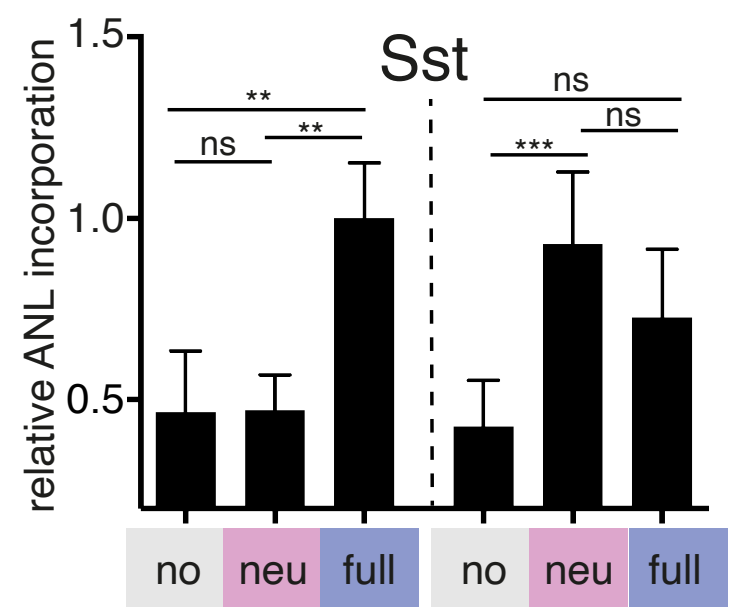


Figure 6. Local protein synthesis occurs in the dendrites of GABAergic neurons.

(A) Schematic outline of the experiment addressing translational capacity of GABAergic neurites (in GAD-Cre::GFP-2A-MetRS*, Sst-Cre::GFP-2A-MetRS*) and glutamatergic neurites (Nex-Cre::GFP-2A-MetRS*) with cell type specific ANL incorporation and BONCAT for results shown in B-E. (B) Representative biotin immunoblot result for an experiment with Sst-Cre::GFP-2A-MetRS* mouse cortical neurons as outlined in A. (C-E) Quantification of the relative biotin western blot signal representing 15 minute ANL incorporation in the soma (left bars) or neurite (right bars) fractions harvested under the conditions shown in $(A)$ from cortical neuron cultures prepared from the indicated mouse lines: (C) GAD-Cre::GFP-2A-MetRS* (D) Sst-Cre::GFP-2A-MetRS* and (E) NexCre::GFP-2A-MetRS*). (mean \pm SD; GAD: soma no $0.6067 \pm 0.07033$, soma neu $0.6533 \pm 0.05538$, soma full $1 \pm 0.07239$, neurites no $0.6800 \pm 0.0600$, neurites neu $0.9617 \pm 0.07360$, neurites full $0.9950 \pm 0.1875$; Sst: soma no $0.4663 \pm 0.1684$, soma neu $0.4706 \pm 0.0980$, soma full $0.9999 \pm 0.1534$, neurites no $0.4271 \pm 0.1271$, neurites neu $0.9290 \pm 0.1996$, neurites full $0.7269 \pm 0.1887$; Nex: soma no $0.7061 \pm 0.1058$, soma neu $0.6814 \pm 0.1011$, soma full $1.000 \pm 0.1167$, neurites no $0.6500 \pm 0.08707$, neurites neu $0.8913 \pm 0.1281$, neurites full $0.9086 \pm 0.1215$ ). Equal protein amounts were loaded within soma fractions and within neurite fractions. The biotin immunoreactivity in the neurite faction of the neurite-only labeling from all investigated mouse lines is significantly higher than in unlabeled controls ((Kruskal-Wallis test with Dunn's multiple comparison's test for GAD $n=6$ (3 experiments); GAD soma: soma no vs soma neu $P>0.9999$, soma no vs soma full $P=0.0032$, soma neu vs soma full $P=0.0304$; $G A D$ neurites: neurites no vs neurites neu $P=0.0239$, neurites no vs neurites full $P=0.0073$, neurites neu vs neurites full $P>0.9999$; Sst $n=7$ (3 experiments); Sst soma: soma no vs soma neu $P>0.9999$, soma no vs soma full $P=0.0023$, soma neu vs soma full $P=0.0089$; Sst neurites: neurites no vs neurites neu $P=0.0008$, neurites no vs neurites full $P=0.0635$, neurites neu vs neurites full $P=0.5449$ ); Nex $n=8$ (4 experiments) Nex soma: soma no vs soma neu $P>0.9999$, soma no vs soma full $P=0.0036$, soma neu vs soma full $P=$ 0.0017 ; Nex neurites: neurites no vs neurites neu $P=0.0050$, neurites no vs neurites full $P=0.0080$, neurites neu vs neurites full $P>0.9999$ ). 
information about its original morphology. Compared to previous attempts at profiling the local transcriptome of single neurons (Middleton et al., 2018; Tóth et al., 2018), our technique dramatically expands the number of cells profiled and mRNAs identified. We discovered that the dendrites of GABAergic neurons contain $~ 4000$ mRNA species, similar to the number observed in glutamatergic neurons. We found the dendritic transcriptome varies according to cell-type, but to a lesser extent than the somatic transcriptome. Additionally, we described the relation between the somatic and dendritic transcriptomes of single neurons and how both the absolute dendritic abundance and enrichment relative to the soma vary according to gene and function. Finally, we demonstrated that, like glutamatergic neurons, GABAergic neurons translate mRNAs in their dendritic compartments.

The subcellular scRNA-seq method presented here combines LCM to isolate cellular compartments from a single neuron, with scRNA-seq to profile the RNAs within it. To decrease the labor-intensive nature of LCM protocols, reduce technical variability, increase sensitivity for low-input samples and enable the use of UMI, we adopted and modified a library preparation method initially developed for droplet microfluidics (Macosko et al., 2015). This allowed us to combine single sub-cellular compartments into fewer samples and use RNA-sequencing to reconstruct the relation between molecules and subcellular compartments. This technique has several limitations. First, our profiles likely represented only a portion of the mRNAs actually present in dendrites. We estimate our method detects mRNA species present in as low as 4 copies in the material collected by LCM, but additional losses likely occur before collection due to RNA degradation and the failure to gather all cut pieces. Accordingly, our dendritic profiles were relatively shallow and may have failed to detect genes present in moderate-to-low copy numbers. This method would thus be inadequate to profile mRNAs within individual dendritic branches. Instead, emerging methods in spatial transcriptomics relying on in-situ hybridization are better equipped for such questions (Eng et al., 2019; Liao et al., 2020; Xia et al., 2019). In contrast to FISH-based transcriptomic techniques, however, our method is unbiased (does not rely on a pre-determined set of transcripts), bypasses 
secondary structure that may limit accessibility for certain mRNAs in situ, and does not suffer from signal saturation of highly abundant transcripts. Therefore, it may be a good option for transcript discovery where a priori information about the sequence or expression levels of transcripts is lacking (e.g. exploring the usage of alternative 3'UTRs or when working in less annotated cell-types or species).

While studies of neuronal mRNA localization have generated several lists of localized mRNAs (see Holt et al., 2019 for a review) much less attention has been paid to potential cell-to-cell diversity. To investigate the role cell identity has on the dendritic transcriptome, we determined the cell types present in our cultures based on the somatic transcriptome. This revealed two types of glutamatergic and three types of GABAergic neurons, although more sub-types likely exist within these categories. We detected some dendritic transcripts that varied according to cell identity. These included some of the genes expressed in a cell-type-specific manner, and, surprisingly, genes expressed at similar levels across different cell types (based on somata transcripts). This latter group suggests differences in the machinery regulating dendritic localization according to cell type. Transport of mRNAs to dendrites is known to rely on interactions between RNA elements, particularly in 3'UTRs, and RNA binding proteins, thus enabling their movement along microtubules (Darnell, 2013; Glock et al., 2017). Cell-type driven variations in mRNA localization could be explained by the cell-type specific expression of proteins capable of modifying RNA elements, like RNA-editing enzymes or epitranscriptome regulators (Behm and Öhman, 2016; Merkurjev et al., 2018), or of RNA binding proteins that may activate dendritic transport of mRNAs containing its targeted elements (Martin and Ephrussi, 2009). Significantly more cell-type specific differences were observed between somata than between dendritic arbors, based on both unsupervised clustering and differential expression analyses. This is to be expected since the soma harbors the dendritic transcriptome in addition to transcripts that maintain cell identity (e.g. transcription factors) and axonal specialization (e.g. neurotransmitter(s) machinery). While it is likely that our method lacks sensitivity, it is also conceivable that less transcriptomic heterogeneity occurs between the dendrites of different neurons for 
biological reasons. Dendrites, as the recipients and integrators of excitatory and inhibitory synaptic inputs, may be more generalized than other cellular compartments. Some celltype-specific dendritic proteins may be synthesized in somata. Additionally, given the complex signal integration and plasticity that occurs within dendrites, is plausible that cell states have bigger influences than cell types in the local mRNA repertoire.

We also investigated the patterns of mRNA distribution between the somatic and dendritic compartments of single neurons. We observed that expression levels established in the soma positively influence the dendritic presence and abundance of some but not all mRNAs, and that the magnitude of this effect significantly varies according to the transcript. By comparing the expression between the dendrites and soma of the same neuron we identified hundreds of genes relatively enriched and de-enriched in dendrites, which occurred at a wide-range of absolute abundances in dendrites. Our results are consistent with a model in which mRNAs compete for access to the transport machinery creating an advantage for the more abundant mRNA species, like Calm1 or Atp5f1b. To compensate, less abundant mRNA species like Ccni and most ribosomal proteins, might increase their affinity for the transport machinery based on the type or number of elements in their sequence. Interestingly, we also observed some mRNAs like Gria2 and Psmb2 that were significantly de-enriched in dendrites despite their moderate-to-high somatic abundances. Although is possible that these mRNAs simply lack the elements that allow dendritic localization, it also possible that mechanisms exist to prevent dendritic entry or to ensure somatic confinement.

Using GO analyses, we identified multiple cellular functions highly represented in the somatic and dendritic transcriptomes. Mitochondrial functions where the most represented among highly abundant somatic and dendritic genes, consistent with the high energetic demands of neuronal compartments. Indeed, local production of mitochondrial proteins was recently implicated in the function of local mitochondria (Kuzniewska et al., 2020), which supply energy to spatially-stable compartments containing multiple synapses (Rangaraju et al., 2019). Intriguingly, we also observed contrasting patterns 
between the protein synthesis and the protein degradation machinery. Ribosomal protein (RP) mRNAs were detected across a wide-range of abundances in both somatic and dendritic compartments, as observed previously (Cajigas et al., 2012; Gumy et al., 2011; Poon et al., 2006). Despite this high variance in absolute expression most RP mRNAs exhibited dendritic enrichments relative to somatic expression of similar magnitudes. In contrast, most proteasome genes were uniformly de-enriched in dendrites despite having diverse somatic expression values. A similar opposing trend in mRNA localization between ribosomal and proteasome genes was observed in the growth cone of cortical projection neurons (Poulopoulos et al., 2019).

Finally, the dendritic mRNAs and the intracellular localization dynamics described above were observed in both GABAergic and Glutamatergic neurons, suggesting both can locally synthesize proteins. Indeed, we observed that GABAergic neurons localize thousands of mRNAs to their dendrites and showed directly that they also perform local translation, suggesting this is a general property of neuronal dendrites. Consistently, a recent study described the local synthesis of protein in an inhibitory axon terminal during plasticity (Younts et al., 2016). Moving forward, it will be important to investigate how plasticity and changes in cell state regulate the dendritic transcriptome of individual neurons, ideally in the in the context of neuronal circuits. For instance, Glutamatergic and GABAergic neuron within a circuit establish an excitatory-inhibitory balance that is crucial for proper brain functions (Nelson and Valakh, 2015). Single cell resolution of the local transcriptome will bring a better understanding of the intracellular mechanisms regulating its properties, as well as better comprehension of the dendritic and axonal compartments participating in diverse neuronal circuits.

\section{Materials and Methods}

\section{Animals}

All animals were housed under conditions approved by the local governmental authorities in standard cages under a 12hr:12hr light-dark cycle with standard lab chow and water 
ad libitum. Female Crl:CD(SD) rats with timed matings were purchased from Charles River Laboratories (Sulzfeld, Germany) (Specific pathogen free (spf) colonies). Newborn rat pups of either sex were used for preparation of hippocampal neuron cultures. Transgenic Cre-driver mouse lines were purchased from the Jackson laboratory (SstIRES-Cre (JAX 013044, (Taniguchi et al., 2011)), GAD2-IRES-Cre (JAX 010802, (Taniguchi et al., 2011))) or kindly provided by K.A. Nave (Nex-Cre (Goebbels et al., 2006)), the conditional STOPflox R26-GFP-2A-MetRS*(L274G) mouse line was developed in house and is available from Jackson Labs ((Alvarez-Castelao et al., 2017), JAX 028071) and all lines were kept under spf conditions. Homozygous females of the Cre-driver lines were crossed to STOPflox R26-GFP-2A-MetRS*(L274G) males and neuron cultures were prepared from cortices and hippocampi of newborn animals without determination of their sex. The procedures involving animal treatment and care were conducted in conformity with the institutional guidelines that are in compliance with the national and international laws and policies (DIRECTIVE2010/63/EU; German animal welfare law, FELASA guidelines) and approved by and reported to the local governmental supervising authorities (Regierungspräsidium Darmstadt). The animals were euthanized according to annex 2 of §2 Abs. 2 Tierschutz-Versuchstier-Verordnung.

\section{Primary neuronal cultures}

Neuronal cultures were prepared and maintained essentially as described (Aakalu et al., 2001). Briefly, the hippocampus of $P 1$ rat pups or cortex and hippocampus of P0/P1 mice of either sex was dissected out, dissociated with papain (Sigma) and plated on poly-Dlysine coated coverslips of MatTek glass bottom dishes (P35G-1.5-14-C) at a density of 20,000 and 30,000 cells/coverslip for LCM and FISH experiments, respectively. For experiments on transwell cell culture inserts mouse cortical neurons were seeded onto 3 $\mu \mathrm{m}$ pore size $75 \mathrm{~mm}$ diameter Polycarbonate membranes (15 Mio) (Costar) or 6-well format PET inserts (1.2 Mio) (Corning) coated with poly-D lysine. All neurons were maintained at $37^{\circ} \mathrm{C}$ and $5 \% \mathrm{CO}_{2}$ in glia- and cortex-conditioned Neurobasal-A (Life Technologies) supplemented with B27 and GlutaMax. Neurons for LCM and FISH experiments were used two weeks and three weeks after plating, respectively. 


\section{Laser Capture Microdissection}

All LCM experiments were conducted with Zeiss's PALM MicroBeam Microscope. Cells were fixed for $5 \mathrm{~min}$ using $70 \%$ ethanol at $-20^{\circ} \mathrm{C}$, and stored at $-80^{\circ} \mathrm{C}$ until day of experiment. Single neurons were selected, imaged and dissected under a 40X objective. Dissection was performed by Laser Pressure Catapulting, with energy of 40, focus of 70 and $15 \%$ of speed. The soma of the neuron was always dissected first, followed by the dendrites. Cells were collected from 12 different plates from 2 separate culture preparations.

\section{Library preparation and sequencing}

Material catapulted by LCM was collected in $3 \mu \mathrm{L}$ of lysis buffer containing: $1 \mathrm{X}$ SingleShot lysis buffer (Bio-rad), $45 \mathrm{mAU} / \mathrm{mL}$ Qiagen Protease (Qiagen), 10mM dNTPs (Thermofisher), $0.1 \mu \mathrm{M}$ of 1 out of 18 different RT custom primers containing an Index and UMI (Table S7), and ERCC RNA standards (Thermo-fisher) diluted 1: $5 \times 10^{6}$. Samples were then incubated at $50^{\circ} \mathrm{C}$ for $10 \mathrm{~min}$ for protein digestion and $75^{\circ} \mathrm{C}$ for $10 \mathrm{~min}$ to inactivate the protease. Reverse transcription (RT) and template switch buffer containing $10 \mathrm{U} / \mu \mathrm{L}$ of SuperScript IV and its buffer at $1 \mathrm{X}$ (Thermo-fisher), 40U/ $\mu \mathrm{L}$ RNAse Inhibitor (Takara Bio), $1 \mu \mathrm{M}$ Template Switch Oligo (Table S7), 6mM MgCl2 (Thermo-fisher), 5mM DTT (Bio-rad) and $1 \mathrm{M}$ betaine (Sigma), was then added to the digestion reaction for a final volume of $6.4 \mu \mathrm{L}$ and incubated at $55^{\circ} \mathrm{C}$ for $10 \mathrm{~min}$ of $\mathrm{RT}$, followed by a $10 \mathrm{~min}$ incubation to inactivate the RT enzyme. Next, samples underwent a pre-amplification PCR reaction containing 1X KAPA Hifi Hot-Star Mix (Roche) and 0.1 $\mu \mathrm{M}$ ISPCR (Table S7) for a final volume of $14 \mu \mathrm{L}$. Then, samples with different indexes but of the same source (somata, dendrites or

empty cuts) were pooled together in groups of 8 and purified using AMPure XP beads (Beckman Coulter). Libraries were further indexed, prepared, and pooled a final time using the Nextera XT DNA library prep kit (Illumina), according to manufacturer's instructions. Library amplification PCR was performed with a custom P5 primer (Table S6). Finally, paired-end sequencing was conducted on the NextSeq 550 (Illumina) according to the following specifications: Read $1=18 \mathrm{bp}$ (Index and UMI), Read $2=58 \mathrm{bp}$ 
(gene), and Read 1 Index = 8bp ( $\mathrm{b}$ index). Read 1 was generated using a custom primer (Table S7). Samples were sequence in four separate runs.

\section{Transcript annotations}

The rat transcriptome is not as well annotated as the mouse or human transcriptome specially in its untranslated regions. This is particularly limiting when using 3'end sequencing (like the subcellular scRNA-seq method used here) to analyze mRNAs localized to neurites since they tend to have longer 3'UTRs that are often unannotated (Tushev et al., 2018). Thus, to improve our detection of mRNAs we used a recent fulllength reconstruction of the hippocampal rat transcriptome using long read sequencing (Wang et al., 2019). We then created a Merged Transcriptome, by combining this fulllength annotation with non-overlapping Ref-seq and Ensemble annotations. Finally, we performed bulk RNA-sequencing on the same plates used for subcellular scRNA-seq and used Cufflinks 2.2.1 (Trapnell et al., 2012) to identify additional alternative 3'UTRs. Cufflinks assembly was done using the Merged Transcriptome as a reference guide and included only additions to genes present in the reference, i.e. novel genes were discarded.

\section{Sequence quality filters, read mapping and generation of digital expression data}

Sequencing data was processed according to the Drop-seq core computation protocol (Macosko et al., 2015). Briefly, using Drop-seq tools (v2.3.0) and Fastp (Chen et al., 2018) reads were removed if they contained low quality indexes or UMI, or low quality, lowcomplexity or too short (<30bp) gene sequences. Additionally, the ends of the reads were trimmed when containing adapter sequences or stretches of $>6$ nt of the same base (eg. Poly A). Reads were aligned to the Rat genome using STAR 2.5.4b. Reads with multiple alignments or excessively soft-clipped (>28 bp) were removed. Reads were then assigned to genes, based on their overlapped with the transcript annotations described above. Finally, UMls of the same gene and same sample (same index), within edit distance of 1 were merged, and counted to determine the expression of each genes within each sample. Mitochondrial encoded mRNAs were excluded from all expression analyses 
as they are locally synthesized and thus beyond the scope of this study. Tables containing mRNA counts per sample were imported into $R$ and analyzed using Seurat 3.1.5.

\section{Data normalization}

All analyses, unless otherwise specified, were performed on data normalized using Seurat's SCTransform function (Hafemeister and Satija, 2019), which uses a negative binomial regression to correct for variation arising from technical factors.

\section{Dimensionality reduction and cluster identification}

Dimensionality reduction consisted of determining the principal components (PC) of the data using Seurat's RunPCA function and then using the top 40 PCs for UMAP embedding using Seurat's RunUMAP function. To identify clusters, we used the top 23 PCs to construct a k-nearest neighbor graph of each cell, which was then used to construct a shared nearest neighbor graph by calculating the overlap between k-nearest neighbors, using Seurat's FindNeighbors function. Modularity was optimized using the Louvain method (Blondel et al., 2008) in Seurat's FindClusters function.

\section{Differential expression}

To determine gene markers of cell-types identified in the somatic dataset (Figure S2A) we used the Seurat FindAllMarkers function to conduct a logistic regression test (Ntranos et al., 2019) on genes expressed in at least $25 \%$ of samples and with a log fold-change above 0.25. A cutoff of adjusted p-value $<0.05$ was used to determine significance. Differential expression analyses between somata or dendrites of different cell types were conducted by a logistic regression test using transcripts detected in at least $19 \%$ of the samples and with a log-fold change of at least 0.95, using Seurat's FindMarkers function (Figure 3A). A cutoff of adjusted $p$-value $<0.05$ was used to determine significance in somata samples. In dendrites, however, this same cutoff reported almost no significant genes in all of the comparisons. To determine whether adjusted p-value of 0.05 was overly punitive when working with a shallower transcriptome and fewer samples (as is the case for dendrites) we performed three iterations of differential expression testing on a 
number of somatic samples equal to dendritic samples, and which were downsampled to equivalent molecular counts (data not shown). In these conditions, an adjusted p-value cutoff of 0.05 missed most somatic differential expression between cell-types. Instead, a p-value of 0.02 preserved most somatic differentially expressed genes among cell types. Thus, we used a p-value of 0.02 as a cutoff for our differential expression analysis between dendrites of different cell types. Paired-differential expression analyses between soma and dendrites of the same neuron were conducted with Seurat's FindMarkers function for genes expressed in at least $25 \%$ of samples using a Poisson generalized linear model (Stuart et al., 2019) with cell of origin as a latent variable. A cutoff of adjusted p-value $<0.05$ was used to determine significance.

\section{Integration with hippocampal tissue scRNA-seq}

Tables containing gene counts per cell were obtained from Zeisel et al., 2015 and Harris et al., 2018 supplementary information, and run through data normalization, dimensionality reduction, cluster identification and differential expression as previously discussed. This allowed us to match the clusters identified in our analysis with those reported by the respective authors. Integration of the two datasets with our somata samples was performed as previously described (Stuart et al., 2019), using canonical correlation analyses to find shared subpopulations across dataset.

\section{Gene ontology analyses}

Gene lists pre-ranked by somatic or dendritic "representation", which equal the product of detection frequency $x$ expression levels for each compartment, were used in a gene set enrichment analysis (GSEA, (Subramanian et al., 2005)), against GO cellular components and SynGO terms (Koopmans et al., 2019). GSEA was performed using a weighted scoring scheme, and only terms containing less than 500 genes and with at least 15 genes present in our data were evaluated.

\section{Immunocytochemistry}


For immunocytochemistry, cells were permeabilized for $15 \mathrm{~min}$ with $0.5 \%$ Triton in blocking buffer (BB) (PBS with 4\% goat serum), blocked in BB for $1 \mathrm{~h}$ and incubated with primary antibodies in $\mathrm{BB}$ for $1 \mathrm{hr}$ at room temperature. After washing, secondary antibodies in BB were applied for 30 min followed, when necessary, by a 3 min incubation with $1 \mu \mathrm{g} / \mu$ l DAPI in PBS to stain nuclei. Cells were washed in PBS and mounted with Aquapolymount (Polysciences).

\section{High sensitivity RNA-FISH}

In situ hybridization was performed using the ViewRNA ISH Cell Assay Kit (Thermofisher) according to the manufacturer's protocol with the modifications described previously (Cajigas et al., 2012). Probe sets targeting the respective mRNAs were purchased from Thermo-fisher. In brief, rat hippocampal or mouse cortical/hippocampal neuron cultures grown on MatTek glass bottom dishes were fixed for 20 min with PBS containing 4\% Sucrose and 4\% PFA, $\mathrm{pH} 7.4$, washed and permeabilized for 5 min with the provided detergent solution. Gene specific type1 and type6 probe sets were applied in 1:100 dilution for $3 \mathrm{hrs}$ at $40^{\circ} \mathrm{C}$. Signal amplification steps with PreAmp/Amp and Label Probe reagents coupled to 550dye (and 650dye or 488dye in dual color FISH) were all performed for $1 \mathrm{hr}$ at $40^{\circ} \mathrm{C}$ with branched DNA reagents diluted in the provided solutions 1:100. Staining for markers was performed after the FISH protocol as described in the immunocytochemistry section starting after the permeabilization step. For experiments in combination with FUNCAT the FISH protocol was performed after the metabolic labeling step and the click reaction applied after finishing the FISH protocol.

\section{Metabolic labeling and FUNCAT}

Mouse neurons expressing the MetRS*(L274G) mutant under control of GAD2-IRES-Cre, Sst-IRES-Cre or Nex-Cre, respectively, were maintained on MatTek glass bottom dishes at $37^{\circ} \mathrm{C}$ and $5 \% \mathrm{CO}_{2}$ in glia- and neuron-conditioned Neurobasal-A with B27 and GlutaMax supplements. For metabolic labeling with ANL the cells were washed for 5 min with Neurobasal A with the above supplements but lacking methionine, incubated for 60 min with $4 \mathrm{mM}$ ANL in Neurobasal $A^{-M e t}$ with supplements and finally washed with their 
own original Methionine-containing medium for $5 \mathrm{~min}$ at $37^{\circ} \mathrm{C} 5 \% \mathrm{CO} 2$ and humidified atmosphere and fixed after another brief washing step as described in the RNA-FISH section. After performing the FISH protocol, the cells were blocked for 30 min with $4 \%$ goat serum in PBS pH7.4, equilibrated to PBS pH7.8 and subjected for 2 hrs to a copper mediated click reaction in PBS pH7.8 at RT (with the following reagents: $200 \mu \mathrm{M}$ Triazole, $500 \mu \mathrm{M}$ TCEP, $2 \mu \mathrm{M}$ Alexa647 alkyne, $200 \mu \mathrm{M}$ CuSO4) before proceeding with immunocytochemistry for marker proteins.

\section{Metabolic labeling of neurites and BONCAT}

Mouse neurons on transwell inserts were maintained at $37^{\circ} \mathrm{C}$ and $5 \% \mathrm{CO}_{2}$ in glia- and cortex-conditioned Neurobasal-A supplemented with B27 and GlutaMax until 10 days in vitro. For the experiment the inserts were carefully rinsed two times in Neurobasal $A$ lacking methionine (Neurobasal $A^{- \text {Met }}$, Life Technologies) and incubated with $8 \mathrm{mM}$ ANL in Neurobasal $A^{-M e t}$ with B27 and Glutamax supplements for 15 min at $37^{\circ} \mathrm{C}$ with $5 \% \mathrm{CO}_{2}$ in a humidified atmosphere with or without prior removal of the soma layer by scraping (above the membrane). Negative control samples were treated in the same way but without ANL added to the media. Soma and neurite layers were harvested separately by two times scraping in PBS, transferred to a microtube followed by a 30 second centrifugation in a benchtop minifuge (neoLab, Heidelberg, Germany). When 6-well transwell membranes were used, material from 3 wells was combined for one replicate sample. After spindown, the supernatant was removed, the pellet immediately frozen on dry ice and stored at $-80^{\circ} \mathrm{C}$ until lysis. The samples were lysed in a minimal volume of PBS with $1 \%(\mathrm{w} / \mathrm{v})$ TritonX-100, $0.4 \%(\mathrm{w} / \mathrm{v})$ SDS, protease inhibitors w/o EDTA (Calbiochem, 1:750) and benzonase (Sigma, 1:1000), heated to $75^{\circ} \mathrm{C}$ for $5 \mathrm{~min}$ and centrifuged. Supernatants were analyzed and adjusted for equal protein content within the soma samples and within the neurite samples of the experiment. BONCAT was performed as described previously (Dieterich et al., 2006). In brief, $20 \mu \mathrm{l}$ of the sample was subjected to a click reaction with $300 \mu \mathrm{M}$ Triazol (Sigma, ref. 678937), $50 \mu \mathrm{M}$ biotinalkyne tag (Thermo-fisher) and $83 \mu \mathrm{g} / \mathrm{mL} \mathrm{CuBr}$ at $4^{\circ} \mathrm{C}$ overnight in the dark in $\mathrm{PBS} \mathrm{pH} 7.8$. SDS-PAGE was performed and proteins were subsequently blotted onto PVDF 
membranes. Biotinylated proteins were detected by Immunoblot with a biotin antibody and IRDye680-coupled secondary antibody and scanned with a fluorescence-based detection system (Odyssey, LICOR, Bad Homburg, Germany).

\section{Western blot analysis}

Corresponding regions-of-interest from the same Western blot were marked in each lane in Fiji ImageJ Version 2.0.0-rc-68/1.52n (NIH), mean grey values were determined for each sample and converted to fraction of the mean value of the full labeling controls in somata for each blot. Biological replicates were from $\geq 3$ independent experiments for each of the 3 mouse lines. Statistical significance of the differences within soma fractions from the different conditions and the neurite fractions from the different conditions were determined for every line by ANOVA (Kruskal-Wallis test corrected for multiple comparisons with Dunn's multiple comparison's test) in GraphPad Prism 6.

\section{ANL synthesis}

ANL was synthesized from Boc-L-Lys-OH (Iris Biotech, Marktredwitz, Germany), Triflic anhydride (trifluoromethanesulfonic acid anhydride, Merck/Sigma and sodium azide in analogy to the synthesis of AHA from Boc-Dab described by(Link et al., 2007).

\section{Antibodies}

The following antibodies were used for immunofluorescence (IF), Immunoblotting (IB) at the indicated dilutions: rabbit anti-biotin (IB 1:1000, Sigma), guinea pig anti-MAP2 (IF 1:1000, Synaptic Systems), anti-rabbit IRDye680 (IB 1:5000, Licor), goat anti-guinea pig Dylight405 (IF 1:500-1:1000, Jackson ImmunoResearch), goat anti-guinea pig-Alexa488 (IF 1:1000, Thermo-fisher).

\section{Confocal microscopy}

Images were acquired with a LSM780 confocal microscope (Zeiss) using a 40x/1.4-NA oil objective (Plan Apochromat 40x/1.4 oil DIC M27) and appropriate laser lines set in single tracks and pixel resolution of at least $2048 \times 2048$. All lasers were used at $2 \%$ power 
or less. Images were acquired in 12-bit or 16bit mode as $z$ stacks with optical slice thickness set to optimal and the detector gain in each channel adjusted to cover the full dynamic range but to avoid saturated pixels. Maximum intensity projections were created in Zen10 software or in Fiji/lmageJ. For visualization, linear adjustments of the single channels for brightness over the whole image were performed. Conditions were kept constant within an experiment.

\section{Soma FISH analysis}

From maximum intensity projections of FISH confocal images somata of all neurons in the field of view were outlined in Fiji, mean grey values were measured in all ROIs representing somata and for each cell mean grey values of cell type marker FISH signal and mRNA of interest FISH signal were plotted in GraphPad Prism 6.

\section{Dendrite FISH pattern visualization with raster plots}

From maximum intensity projections of FISH confocal images dendrites from all neurons in a field of view that could be followed for a minimum length of around $50 \mu \mathrm{m}$ were traced in Image J/Fiji) along the MAP2 label with the segmented line tool starting at the soma until the most distal point where the dendrite was identified without crossing other cells containing signal. Dendrites were straightened, channels split and converted in profiles of mean intensity values. For each dendrite, signal puncta were detected as peaks in the profile and converted into bar signals at the corresponding location in a representation of the dendrite with a custom MatLab script. All analyzed dendrite/signal bar representations were stacked in a soma (left) to distal (right) direction to visualize variability and distribution pattern with the end of the tracked dendrite marked in the plot. In Fig 3 dendrites were sorted prior to plotting based on a threshold level in a second channel stained for the cell type marker mRNAs Gad1/2. For FISH experiments in Fig 4F, neuron identity was tracked by cell-type specific ANL incorporation into mouse cortical neurons (from mouse lines described in Fig 6) and FUNCAT. To ensure coverage of excitatory and inhibitory neuron dendrites in the raster plots, cells with and without FUNCAT signal were imaged and dendrites straightened. 


\section{Acknowledgements}

We thank Ina Bartnik, Dirk Vogel, Bernadette Tune, Matt White and Michelle Gottlieb for assistance with experiments and analyses, Georgi Tushev for help with FISH analyses, Or Shahar and Stephan Junek for help with the LCM microscope, and Belquis NassimAssir for help with BONCAT experiments. B.A.C is funded by Comunidad de Madrid (Atracción de Talento-2019T1/BMD-14057) and Ministerio de Ciencia e Innovación (Ramón y Cajal- RYC2018-024435-I). E.M.S. is funded by the Max Planck Society, an Advanced Investigator award from the European Research Council (grant 743216), DFG CRC 1080: Molecular and Cellular Mechanisms of Neural Homeostasis, and DFG CRC 902: Molecular Principles of RNA-based Regulation.

\section{Competing interests}

The authors declare no competing interests.

\section{Author Contributions}

J.D.P. and E.M.S designed the study and wrote the paper. J.D.P. developed the Subcellular scRNA-seq protocol, performed sequencing experiments and analyzed the data. I.C.W.C. assisted in the development of the protocol. S.t.D performed and analyzed FISH and metabolic labeling experiments. B.A.C. performed and analyzed BONCAT experiments.

\section{Data Availability}

The accession number for the raw sequencing data reported in this paper is GEO: GSE157204. Metadata with information about the scRNA-seq experimental designed and quality metrics are included in Table S1. scRNA-seq processed data used for analyses in this manuscript is included in Table S2.

\section{Tables}


Table S1. Sample Metadata. Related to figures 1-4. Contains information on the experimental design, quality metrics, and experimental variables for each Subcellular scRNA-seq sample.

Table S2. Count data. Related to figures 1-4. Contains UMI counts in a genes (rows) by samples (columns) table.

Table S3. Somatic and dendritic transcriptomes of GABAergic and glutamatergic neurons. Related to figure 2. Contains average expression values of genes detected in the somata and dendrites of GABAergic and glutamatergic neurons.

Table S4. Somata and dendrites cell-type-specific differential expression results. Related to figure 3. Contains results of differential expression test for: (Tab 1) GABAergic vs Glutamatergic Somata, (Tab 2) GABAergic vs Glutamatergic Dendrites, (Tab 3) GABAergic 1 vs GABAergic 2 \& 3 Somata, (Tab 4) GABAergic 1 vs GABAergic 2 \& 3 Dendrites, (Tab 5) GABAergic 2 vs GABAergic 3 Somata, (Tab 6) GABAergic 1 vs GABAergic 2 \& 3 Dendrites.

Table S5. Somata vs dendrites differential expression results. Related to figure 4. Contains results of somata vs dendrites differential expression test.

Table S6. Gene Ontology Analysis. Related to figure 5. Contains results of GSEA ranked test for somata (Tab 1) and dendrites (Tab 2).

Table S7. Sequences of primers used in this study. Related to methods.

\section{References}

Aakalu, G., Smith, W.B., Nguyen, N., Jiang, C., Schuman, E.M., 2001. Dynamic visualization of local protein synthesis in hippocampal neurons. Neuron 30, 489502. doi:10.1016/s0896-6273(01)00295-1 
Alvarez-Castelao, B., Schanzenbächer, C.T., Hanus, C., Glock, C., Tom Dieck, S., Dörrbaum, A.R., Bartnik, I., Nassim-Assir, B., Ciirdaeva, E., Mueller, A., Dieterich, D.C., Tirrell, D.A., Langer, J.D., Schuman, E.M., 2017. Cell-type-specific metabolic labeling of nascent proteomes in vivo. Nature Biotechnology 35, 1196-1201. doi:10.1038/nbt.4016

Alvarez-Castelao, B., Schanzenbächer, C.T., Langer, J.D., Schuman, E.M., 2019. Celltype-specific metabolic labeling, detection and identification of nascent proteomes in vivo. Nature protocols 14, 556-575. doi:10.1038/s41596-018-0106-6

Alvarez-Castelao, B., Tom Dieck, S., Fusco, C.M., Donlin-Asp, P., Perez, J.D., Schuman, E.M., 2020. The switch-like expression of heme-regulated kinase 1 mediates neuronal proteostasis following proteasome inhibition. eLife 9. doi:10.7554/eLife.52714

Behm, M., Öhman, M., 2016. RNA Editing: A Contributor to Neuronal Dynamics in the Mammalian Brain. Trends Genet 32, 165-175. doi:10.1016/j.tig.2015.12.005

Bingol, B., Schuman, E.M., 2006. Activity-dependent dynamics and sequestration of proteasomes in dendritic spines. Nature 441, 1144-1148. doi:10.1038/nature04769

Blondel, V.D., Guillaume, J.-L., Lambiotte, R., Lefebvre, E., 2008. Fast unfolding of communities in large networks. J. Stat. Mech. 2008, P10008-14. doi:10.1088/17425468/2008/10/P10008

Butler, A., Hoffman, P., Smibert, P., Papalexi, E., Satija, R., 2018. Integrating single-cell transcriptomic data across different conditions, technologies, and species. Nature Biotechnology 36, 411-420. doi:10.1038/nbt.4096

Cajigas, I.J., Tushev, G., Will, T.J., Tom Dieck, S., Fuerst, N., Schuman, E.M., 2012. The Local Transcriptome in the Synaptic Neuropil Revealed by Deep Sequencing and High-Resolution Imaging. Neuron 74, 453-466. doi:10.1016/j.neuron.2012.02.036

Chen, S., Zhou, Y., Chen, Y., Gu, J., 2018. fastp: an ultra-fast all-in-one FASTQ preprocessor. Bioinformatics 34, i884-i890. doi:10.1093/bioinformatics/bty560

Darnell, R.B., 2013. RNA protein interaction in neurons. Annu. Rev. Neurosci. 36, 243270. doi:10.1146/annurev-neuro-062912-114322

Dieterich, D.C., Lee, J.J., Link, A.J., Graumann, J., Tirrell, D.A., Schuman, E.M., 2007. Labeling, detection and identification of newly synthesized proteomes with bioorthogonal non-canonical amino-acid tagging. Nature protocols 2, 532-540. doi:10.1038/nprot.2007.52

Eng, C.-H.L., Lawson, M., Zhu, Q., Dries, R., Koulena, N., Takei, Y., Yun, J., Cronin, C., Karp, C., Yuan, G.-C., Cai, L., 2019. Transcriptome-scale super-resolved imaging in tissues by RNA seqFISH. Nature 1-24. doi:10.1038/s41586-019-1049-y

Foley, J.W., Zhu, C., Jolivet, P., Zhu, S.X., Lu, P., Meaney, M.J., West, R.B., 2019. Gene expression profiling of single cells from archival tissue with laser-capture microdissection and Smart-3SEQ. Genome Research 29, 1816-1825. doi:10.1101/gr.234807.118

Glock, C., Biever, A., Tushev, G., Bartnik, I., bioRxiv, B.N.-A., 2020, n.d. The mRNA translation landscape in the synaptic neuropil. biorxiv.org . doi:10.1021/jp104731s 
Glock, C., Heumüller, M., Schuman, E.M., 2017. mRNA transport \& local translation in neurons. Curr Opin Neurobiol 45, 169-177. doi:10.1016/j.conb.2017.05.005

Goebbels, S., Bormuth, I., Bode, U., Hermanson, O., Schwab, M.H., Nave, K.-A., 2006. Genetic targeting of principal neurons in neocortex and hippocampus of NEX-Cre mice. Genesis 44, 611-621. doi:10.1002/dvg.20256

Gumy, L.F., Yeo, G.S.H., Tung, Y.-C.L., Zivraj, K.H., Willis, D., Coppola, G., Lam, B.Y.H., Twiss, J.L., Holt, C.E., Fawcett, J.W., 2011. Transcriptome analysis of embryonic and adult sensory axons reveals changes in mRNA repertoire localization. RNA 17, 85-98. doi:10.1261/rna.2386111

Hafemeister, C., Satija, R., 2019. Normalization and variance stabilization of single-cell RNA-seq data using regularized negative binomial regression. Genome Biol 20, 296-15. doi:10.1186/s13059-019-1874-1

Hafner, A.-S., Donlin-Asp, P.G., Leitch, B., Herzog, E., Schuman, E.M., 2019. Local protein synthesis is a ubiquitous feature of neuronal pre- and postsynaptic compartments. Science 364, eaau3644. doi:10.1126/science.aau3644

Hanus, C., Geptin, H., Tushev, G., Garg, S., Alvarez-Castelao, B., Sambandan, S., Kochen, L., Hafner, A.-S., Langer, J.D., Schuman, E.M., 2016. Unconventional secretory processing diversifies neuronal ion channel properties. eLife 5, 74. doi:10.7554/eLife.20609

Harris, K.D., Hochgerner, H., Skene, N.G., Magno, L., Katona, L., Bengtsson Gonzales, C., Somogyi, P., Kessaris, N., Linnarsson, S., Hjerling-Leffler, J., 2018. Classes and continua of hippocampal CA1 inhibitory neurons revealed by single-cell transcriptomics. Plos Biol 16, e2006387. doi:10.1371/journal.pbio.2006387

Holt, C.E., Martin, K.C., Schuman, E.M., 2019. Local translation in neurons: visualization and function. Nature Structural \& Molecular Biology 26, 557-566. doi:10.1038/s41594-019-0263-5

Huang, Z.J., Paul, A., 2019. The diversity of GABAergic neurons and neural communication elements. Nat Rev Neurosci 20, 563-572. doi:10.1038/s41583-0190195-4

Koopmans, F., van Nierop, P., Andres-Alonso, M., Byrnes, A., Cijsouw, T., Coba, M.P., Cornelisse, L.N., Farrell, R.J., Goldschmidt, H.L., Howrigan, D.P., Hussain, N.K., Imig, C., de Jong, A.P.H., Jung, H., Kohansalnodehi, M., Kramarz, B., Lipstein, N., Lovering, R.C., MacGillavry, H., Mariano, V., Mi, H., Ninov, M., Osumi-Sutherland, D., Pielot, R., Smalla, K.-H., Tang, H., Tashman, K., Toonen, R.F.G., Verpelli, C., Reig-Viader, R., Watanabe, K., van Weering, J., Achsel, T., Ashrafi, G., Asi, N., Brown, T.C., Pietro De Camilli, Feuermann, M., Foulger, R.E., Gaudet, P., Joglekar, A., Kanellopoulos, A., Malenka, R., Nicoll, R.A., Pulido, C., de Juan-Sanz, J., Sheng, M., Südhof, T.C., Tilgner, H.U., Bagni, C., Bayés, À., Biederer, T., Brose, N., Chua, J.J.E., Dieterich, D.C., Gundelfinger, E.D., Hoogenraad, C., Huganir, R.L., Jahn, R., Kaeser, P.S., Kim, E., Kreutz, M.R., McPherson, P.S., Ben M Neale, O'Connor, V., Posthuma, D., Ryan, T.A., Sala, C., Feng, G., Hyman, S.E., Thomas, P.D., Smit, A.B., Verhage, M., 2019. SynGO: An Evidence-Based, Expert-Curated Knowledge Base for the Synapse. Neuron 103, 217-234.e4.

doi:10.1016/j.neuron.2019.05.002 
Kosik, K.S., 2016. Life at Low Copy Number: How Dendrites Manage with So Few mRNAs. Neuron 92, 1168-1180. doi:10.1016/j.neuron.2016.11.002

Kuzniewska, B., Cysewski, D., Wasilewski, M., Sakowska, P., Milek, J., Kulinski, T.M., Winiarski, M., Kozielewicz, P., Knapska, E., Dadlez, M., Chacinska, A., Dziembowski, A., Dziembowska, M., 2020. Mitochondrial protein biogenesis in the synapse is supported by local translation. EMBO Rep e48882. doi:10.15252/embr.201948882

Kügelgen, N., Chekulaeva, M., 2020. Conservation of a core neurite transcriptome across neuronal types and species. WIREs RNA 9, 2579-16.

doi:10.1002/wrna.1590

Liao, J., Lu, X., Shao, X., Zhu, L., Fan, X., 2020. Uncovering an Organ's Molecular Architecture at Single-Cell Resolution by Spatially Resolved Transcriptomics.

Trends Biotechnol. 1-16. doi:10.1016/j.tibtech.2020.05.006

Link, A.J., Vink, M.K.S., Tirrell, D.A., 2007. Preparation of the functionalizable methionine surrogate azidohomoalanine via copper-catalyzed diazo transfer. Nature protocols 2, 1879-1883. doi:10.1038/nprot.2007.268

Liu, Y., Beyer, A., Aebersold, R., 2016. On the Dependency of Cellular Protein Levels on mRNA Abundance. Cell 165, 535-550. doi:10.1016/j.cell.2016.03.014

Macosko, E.Z., Basu, A., Satija, R., Nemesh, J., Shekhar, K., Goldman, M., Tirosh, I., Bialas, A.R., Kamitaki, N., Martersteck, E.M., Trombetta, J.J., Weitz, D.A., Sanes, J.R., Shalek, A.K., Regev, A., Mccarroll, S.A., 2015. Highly Parallel Genome-wide Expression Profiling of Individual Cells Using Nanoliter Droplets. Cell 161, 12021214. doi:10.1016/j.cell.2015.05.002

Martin, K.C., Ephrussi, A., 2009. mRNA Localization: Gene Expression in the Spatial Dimension. Cell 136, 719-730. doi:10.1016/j.cell.2009.01.044

Merkurjev, D., Hong, W.-T., lida, K., Oomoto, I., Goldie, B.J., Yamaguti, H., Ohara, T., Kawaguchi, S.-Y., Hirano, T., Martin, K.C., Pellegrini, M., Wang, D.O., 2018. Synaptic N 6 -methyladenosine (m 6 A) epitranscriptome reveals functional partitioning of localized transcripts. Nat Neurosci 21, 1004-1014.

doi:10.1038/s41593-018-0173-6

Middleton, S.A., Eberwine, J., Kim, J., 2018. Comprehensive catalog of dendritically localized mRNA isoforms from sub-cellular sequencing of single mouse neurons. bioRxiv 1-41. doi:10.1101/278648

Nelson, S.B., Valakh, V., 2015. Excitatory/Inhibitory Balance and Circuit Homeostasis in Autism Spectrum Disorders. Neuron 87, 684-698.

doi:10.1016/j.neuron.2015.07.033

Nichterwitz, S., Chen, G., Benitez, J.A., Yilmaz, M., Storvall, H., Cao, M., Sandberg, R., Deng, Q., Hedlund, E., 2016. Laser capture microscopy coupled with Smart-seq2 for precise spatial transcriptomic profiling. Nat Comms 7, 12139.

doi:10.1038/ncomms12139

Ntranos, V., Yi, L., Melsted, P., Pachter, L., 2019. A discriminative learning approach to differential expression analysis for single-cell RNA-seq. Nature Publishing Group 18. doi:10.1038/s41592-018-0303-9 
Pelkey, K.A., Chittajallu, R., Craig, M.T., Tricoire, L., Wester, J.C., McBain, C.J., 2017. Hippocampal GABAergic Inhibitory Interneurons. Physiological Reviews 97, 16191747. doi:10.1152/physrev.00007.2017

Poon, M.M., Choi, S.-H., Jamieson, C.A.M., Geschwind, D.H., Martin, K.C., 2006. Identification of process-localized mRNAs from cultured rodent hippocampal neurons. Journal of Neuroscience 26, 13390-13399.

doi:10.1523/JNEUROSCI.3432-06.2006

Poulopoulos, A., Murphy, A.J., Ozkan, A., Davis, P., Hatch, J., Kirchner, R., Macklis, J.D., 2019. Subcellular transcriptomes and proteomes of developing axon projections in the cerebral cortex. Nature 1-24. doi:10.1038/s41586-018-0847-y

Prakadan, S.M., Shalek, A.K., Weitz, D.A., 2017. Scaling by shrinking: empowering single-cell "omics" with microfluidic devices. Nat Rev Genet 18, 345-361. doi:10.1038/nrg.2017.15

Rangaraju, V., Lauterbach, M., Schuman, E.M., 2019. Spatially Stable Mitochondrial Compartments Fuel Local Translation during Plasticity. Cell 176, 73-84.e15. doi:10.1016/j.cell.2018.12.013

Saunders, A., Macosko, E.Z., Wysoker, A., Goldman, M., Krienen, F.M., de Rivera, H., Bien, E., Baum, M., Bortolin, L., Wang, S., Goeva, A., Nemesh, J., Kamitaki, N., Brumbaugh, S., Kulp, D., Mccarroll, S.A., 2018. Molecular Diversity and Specializations among the Cells of the Adult Mouse Brain. Cell 174, 10151030.e16. doi:10.1016/j.cell.2018.07.028

Stuart, T., Butler, A., Hoffman, P., Hafemeister, C., Papalexi, E., Mauck, W.M., III, Hao, Y., Stoeckius, M., Smibert, P., Satija, R., 2019. Comprehensive Integration of Single-Cell Data. Cell 177, 1888-1902.e21. doi:10.1016/j.cell.2019.05.031

Subramanian, A., Tamayo, P., Mootha, V.K., Mukherjee, S., Ebert, B.L., Gillette, M.A., Paulovich, A., Pomeroy, S.L., Golub, T.R., Lander, E.S., Mesirov, J.P., 2005. Gene set enrichment analysis: a knowledge-based approach for interpreting genome-wide expression profiles. Proc Natl Acad Sci USA 102, 15545-15550.

doi:10.1073/pnas.0506580102

Taniguchi, H., He, M., Wu, P., Kim, S., Paik, R., Sugino, K., Kvitsiani, D., Kvitsani, D., Fu, Y., Lu, J., Lin, Y., Miyoshi, G., Shima, Y., Fishell, G., Nelson, S.B., Huang, Z.J., 2011. A resource of Cre driver lines for genetic targeting of GABAergic neurons in cerebral cortex. Neuron 71, 995-1013. doi:10.1016/j.neuron.2011.07.026

Tóth, E.N., Lohith, A., Mondal, M., Guo, J., Fukamizu, A., Pourmand, N., 2018. Singlecell nanobiopsy reveals compartmentalization of mRNAs within neuronal cells. J Biol Chem 293, 4940-4951. doi:10.1074/jbc.M117.800763

Trapnell, C., Roberts, A., Goff, L., Pertea, G., Kim, D., Kelley, D.R., Pimentel, H., Salzberg, S.L., Rinn, J.L., Pachter, L., 2012. Differential gene and transcript expression analysis of RNA-seq experiments with TopHat and Cufflinks. Nature protocols 7, 562-578. doi:10.1038/nprot.2012.016

Tushev, G., Glock, C., Heumüller, M., Biever, A., Jovanovic, M., Schuman, E.M., 2018. Alternative 3' UTRs Modify the Localization, Regulatory Potential, Stability, and Plasticity of mRNAs in Neuronal Compartments. Neuron 98, 495-511.e6. doi:10.1016/j.neuron.2018.03.030 
Wang, X., You, X., Langer, J.D., Hou, J., Rupprecht, F., Vlatkovic, I., Quedenau, C., Tushev, G., Epstein, I., Schaefke, B., Sun, W., Fang, L., Li, G., Hu, Y., Schuman, E.M., Chen, W., 2019. Full-length transcriptome reconstruction reveals a large diversity of RNA and protein isoforms in rat hippocampus. Nat Comms 1-15. doi:10.1038/s41467-019-13037-0

Xia, C., Fan, J., Emanuel, G., Hao, J., Zhuang, X., 2019. Spatial transcriptome profiling by MERFISH reveals subcellular RNA compartmentalization and cell cycledependent gene expression. Proc Natl Acad Sci USA 116, 19490-19499. doi:10.1073/pnas.1912459116

Younts, T.J., Monday, H.R., Dudok, B., Klein, M.E., Jordan, B.A., Katona, I., Castillo, P.E., 2016. Presynaptic Protein Synthesis Is Required for Long-Term Plasticity of GABA Release. Neuron 92, 479-492. doi:10.1016/j.neuron.2016.09.040

Zeisel, A., Hochgerner, H., Lönnerberg, P., Johnsson, A., Memic, F., van der Zwan, J., Häring, M., Braun, E., Borm, L.E., La Manno, G., Codeluppi, S., Furlan, A., Lee, K., Skene, N., Harris, K.D., Hjerling-Leffler, J., Arenas, E., Ernfors, P., Marklund, U., Linnarsson, S., 2018. Molecular Architecture of the Mouse Nervous System. Cell 174, 999-1014.e22. doi:10.1016/j.cell.2018.06.021

Zeisel, A., Muñoz-Manchado, A.B., Codeluppi, S., Lönnerberg, P., La Manno, G., Juréus, A., Marques, S., Munguba, H., He, L., Betsholtz, C., Rolny, C., CasteloBranco, G., Hjerling-Leffler, J., Linnarsson, S., 2015. Cell types in the mouse cortex and hippocampus revealed by single-cell RNA-seq. Science 347, 1138-1142. doi:10.1126/science.aaa1934

Zhong, J., Zhang, T., Bloch, L.M., 2006. Dendritic mRNAs encode diversified functionalities in hippocampal pyramidal neurons. BMC Neurosci 7, 17.

doi:10.1186/1471-2202-7-17 